\title{
Heat Stress Impact and Genetic Diversity among Some Bread Wheat Genotypes
}

\author{
A. Hamda and K. A. M. Ibrahim* \\ Agronomy Department, Faculty of Agriculture, Assiut \\ University, Assiut and *Agronomy Department, Faculty of \\ Agriculture, Assiut University, New Valley, Egypt.
}

\begin{abstract}
WHEAT is the most widely grown crop in the world especially in the developing countries. Recently, the climatological extremes including high temperatures is predicted to have a general negative effect on wheat production due to the damaging effect on plant development especially during anthesis stage. Heat tolerance is a complex trait and influenced by different components. A panel of 40 wheat genotypes evaluated for 8 yield and yield-contributing traits under recommended sowing date of the Egyptian Ministry of Agriculture as a control and two other different sowing dates as plants will face heat-stressed conditions at anthesis and grain-filling phases. All measured phenotypic traits exhibited highly significant differences both among evaluated accessions and sowing dates in both growing seasons. A continuous phenotypic variation in all measured traits found, indicating a polygenic inheritance of measured traits. The ANOVA revealed highly significant genotype $\times$ environment interaction which is expected for quantitative traits. Cluster analysis revealed two distinct groups with respect to stress tolerance index with substantial diversity among genotypes either susceptible or tolerant to heat stress. Cophenetic correlation between ultrametric similarities of tree and similarity matrix was found to be relatively high $(\mathrm{r}=0.76, \mathrm{P}$ $<0.01$ ), suggesting that the cluster analysis strongly represents the similarity matrix.
\end{abstract}

Keywords: Wheat, Triticum, Heat stress, Cluster analysis.

A substantial increase in world food supply of $70-100 \%$ is required to feed the expected 9 billon world population in 2050 (Godfray et al., 2010). Because of limited possibilities to extend existing crop-growing areas, a substantial increase in crop productivity is essential to guarantee future food security (Parry et al., 2011 and Reynolds et al., 2011). Wheat is one of the most widely grown cereal crops, consumed as food, with an average of 53\% in the developed world and close to $85 \%$ in the developing countries (FAO, 2014). This widely grown possesses some adaptive resilience which is the ability to display some phenotypic changes in responses to environmental conditions. High temperatures at the end of wheat-growing season in Mediterranean climate regions like Egypt are a major abiotic stress affecting yield and its components (Joshi et al., 2007 and Said et al., 2015). The consequences of climate changes are likely to include continuous increase in ambient temperatures and hence an increase in the frequency and extent of heat stress periods in the near future for the 
Mediterranean. Therefore, high temperature is considered as a key stress factor with high potential impact on crop yield (Suzuki et al., 2014). Such increases in temperature can cause heat stress which as a great threat to wheat production in many developing countries, particularly when it occurs during reproductive and grain-filling phases (Fischer \& Byerlee, 1991).

Heat tolerance is a complex quantitative trait involved epistatic interactions among loci and strong genotype $\times$ environment interactions and not correlated to a single 'thermotolerant' gene in cereals (Maestri et al., 2002) and understanding the genetic basis of tolerance to high temperature is important for improving the productivity of wheat (Triticum aestivum L.) particularly under heat stress regions (Yang et al., 2002). The impact of high temperature stress is a complex function of intensity, duration, and rate of temperature change. High temperature stress can alter multiple aspects of cellular physiology such as membrane fluidity, nucleic acid and protein structures, as well as metabolite and osmolyte concentrations (Wahid et al., 2007 and Wang et al., 2003). Heat shock proteins, low-molecularweight proteins, are generally produced only in response to environmental stress particularly high temperature. These proteins have been reported to have a role in stabilizing the structure of other proteins and have been reported to serve as molecular chaperones that participate in adenosine triphosphate-dependent protein unfolding or assembly/disassembly reactions and prevent protein denaturation during stress (Gorantla et al., 1984 and Wahid et al., 2007).

Stress tolerance can be improved by selecting and developing wheat genotypes with heat resistance. Improvement in grain yield under heat stress implies selecting genotypes for grain size (Farooq et al., 2011; Fokar et al., 1998 and Wardlaw et al., 2002). Therefore, establishment of breeding strategies is of great importance in the development of cultivars with improved tolerance to climatic and environmental changes (Stratonovitch \& Semenov, 2015). By understanding of the causes of genotypic-environment interaction we can stablish breeding objectives, identify ideal test conditions, and formulate recommendations for areas of optimal cultivar adaptation (Weikai \& Hunt, 2001).

The potential goal of the current study is to screen the primary gene pool of wheat to identify heat tolerant genotypes as an initial step towards devolving heat stress tolerant cultivars.

\section{Materials and Methods}

\section{Plant Material and experiments}

A panel of 40 bread wheat genotypes (Triticum aestivum) were included in the current study (18 local cultivars, 6 imported cultivars, 5 promising inbred lines developed in Agronomy Department Assiut University and 11 landraces) (Table 1). Three experiments were conducted in clay soil type with $\mathrm{pH} 7.73$ and 7.8 in 2013/2014 and 2014/2015 growing seasons, respectively, at Assiut University Experimental Farm (lat $27^{\circ} 03^{\prime} \mathrm{N}$, long $31^{\circ} 01^{\prime}$ and alt $70 \mathrm{~m}$ asl). Each experiment consists of 40 genotypes which did not differ significantly in heading dates (data not shown) were grown in the field with plant to plant distance of $15 \mathrm{~cm}$

Egypt. J. Agron. 38, No. 3 (2016) 
and row to row distance of $20 \mathrm{~cm}$ with 3 replications (Each replication consisted of one nursery row $3 \mathrm{~m}$ in length. Genotypes were sown in three sowing dates: i) recommended sowing date of the Egyptian Ministry of Agriculture in upper Egypt as the favorable sowing date (FSD) on Dec. 1, 2013 and Dec. 3, 2014 (where plants will not face high temperature during anthesis and grain-filling phases by the end of Feb.), ii) the moderately heat-stressed sowing date (MHSD) on Jan. 1, 2013, and Jan. 6, 2014 (where plants have experienced terminal high temperature during anthesis and grain-filling phases by the end of March) and iii) the severely heatstressed sowing date (SHSD) on Feb. 1, 2013 and Feb. 9, 2014 (where plants have experienced terminal high temperature during anthesis and grain-filling phases by the middle of April ) (Table 2). The experiments were fully fertilized and well service irrigated (6 irrigations every 21 days intervals) to avoid any nutritional or water deficit stress according to the recommended treatments in the experiment region. It rarely to have rain in the experimental site as it rained only one time on Mar. $9^{\text {th }} 2014$ and we skipped the irrigation of this month and there were no rains in 2014/2015season. By the end of May after maturity, a sample of 3 guarded plants from each row was harvested then kept in green house till well dry in the two growing seasons.

\section{Phenotypic evaluation}

Phenotypic data was assessed on 8 agronomic traits including; plant height in $\mathrm{cm}$ (PH; height of main stem at maturity), biological (above-ground dry matter) yield per plant in gram (BY), weight grain yield per plant in gram (GY), harvest index (HI), spike length in $\mathrm{cm}$ (SL; length from neck node to the tip of spike at maturity), number of spikes per plant (SN), spikelet number per spike (SNS) and 100 kernel weight in gram $(100 \mathrm{KW})$. In addition, the average over all the tow growing seasons of stress tolerance index (STI) was calculated for all studied traits following the formula described by Fernandez (1992) as $\mathrm{STI}=\frac{\mathrm{Ys} * \mathrm{Yp}}{\overline{\mathrm{Y}} \mathrm{p}^{2}}$ where Ys and $\mathrm{Yp}$ are severe stress and non-stress potential yield of a given genotype, respectively. $\overline{\mathrm{Y}} \mathrm{p}$ is the average yield of all genotypes under nonstress conditions (Table 3 ).

\section{Statistical analysis}

Randomized complete block design combined over sowing dates and seasons was used with three replications for a genotype in each planting date. Analysis of variance (ANOVA) was carried out using Proc Mixed of SAS package version 9.2 (SAS 2008) and means were compared by revised Least Significant Difference (LS D') at 5\% level of significant (Steel \& Torrie, 1981). For cluster analysis, stress tolerance index (STI) was used. The Euclidean distance matrix with un-weighted pair-group method based on arithmetic averages (UPGMA) in the software NTSYS-pc ver 2.1 (Rohlf, 2000) was implemented to develop a dendogram. The output was analyzed using an agglomerative hierarchical clustering method with complete linkage strategy. Data was subjected to analysis to produce a matrix of dissimilarity values and the phenotypic distance between each pair of accessions was estimated as Euclidean distance. 
TABLE 1. List of the 40 genotypes and its origin.

\begin{tabular}{|c|c|c|}
\hline Type & Originated location & Name \\
\hline \multirow{18}{*}{ 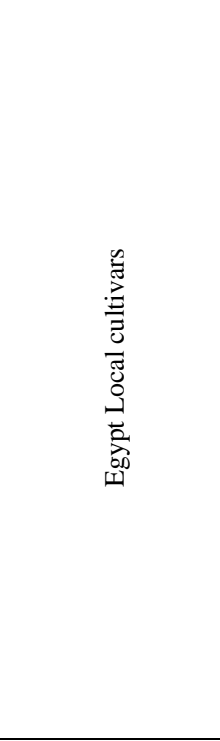 } & \multirow{4}{*}{$\begin{array}{c}\text { Originated from Regional } \\
\text { Agricultural Research Station in } \\
\text { northern Delta }\end{array}$} & Sakha 8 \\
\hline & & Sakha 69 \\
\hline & & Sakha 92 \\
\hline & & Sakha 93 \\
\hline & \multirow{4}{*}{$\begin{array}{c}\text { Originated from Regional } \\
\text { Agricultural Research Station in } \\
\text { Central Delta }\end{array}$} & Gemmeza 7 \\
\hline & & Gemmeza 10 \\
\hline & & Misr 1 \\
\hline & & Misr 2 \\
\hline & \multirow{4}{*}{$\begin{array}{l}\text { Originated from Agricultural } \\
\text { Research Station in Giza }\end{array}$} & Giza 160 \\
\hline & & Giza 165 \\
\hline & & Giza 168 \\
\hline & & Giza 171 \\
\hline & \multirow{4}{*}{$\begin{array}{c}\text { Originated from Regional } \\
\text { Research Station of Central Egypt }\end{array}$} & Sids 1 \\
\hline & & Sids 4 \\
\hline & & Sids 13 \\
\hline & & Sids 12 \\
\hline & \multirow{2}{*}{$\begin{array}{c}\text { Originated from Regional } \\
\text { Research Station of Upper Egypt }\end{array}$} & Sahel 1 \\
\hline & & Shandaweel 1 \\
\hline \multirow{2}{*}{\multicolumn{2}{|c|}{ Imported from Canada }} & Canada 462 \\
\hline & & Canada 515 \\
\hline \multirow{4}{*}{\multicolumn{2}{|c|}{ Imported from Sudan }} & Beknora \\
\hline & & Debera \\
\hline & & Nelen \\
\hline & & Snora \\
\hline \multirow{5}{*}{$\begin{array}{l}\text { Agronomy } \\
\text { Department Assiut } \\
\text { University, Egypt }\end{array}$} & \multirow{5}{*}{ Promising inbred lines } & Assiut 108 \\
\hline & & Assiut 204 \\
\hline & & Assiut 724 \\
\hline & & MK 1-20 \\
\hline & & MK 7-83 \\
\hline & \multirow{11}{*}{ Landraces } & L 1203 \\
\hline & & L 1290 \\
\hline & & L 1351 \\
\hline & & L 1457 \\
\hline & & L 741 \\
\hline & & L 780 \\
\hline & & L 887 \\
\hline & & line 1 \\
\hline & & line 3 \\
\hline & & line 4 \\
\hline & & line 5 \\
\hline
\end{tabular}

Egypt. J. Agron. 38, No. 3 (2016) 
TABLE 2. Assiut monthly average of minimum (Min), average of maximum (Max) and general mean of air temperature $\left({ }^{\circ} \mathrm{C}\right)$ during the two growing seasons 2013/2014 and 2014/2015.

\begin{tabular}{|l|c|c|c|c|c|c|}
\hline \multirow{2}{*}{ Month } & \multicolumn{2}{|c|}{ Min. Temperature } & \multicolumn{2}{c|}{ Max. Temperature } & \multicolumn{2}{c|}{ General mean } \\
\cline { 2 - 7 } & $\mathbf{2 0 1 3 / 2 0 1 4}$ & $\mathbf{2 0 1 4 / 2 0 1 5}$ & $\mathbf{2 0 1 3 / 2 0 1 4}$ & $\mathbf{2 0 1 4 / 2 0 1 5}$ & $\mathbf{2 0 1 3 / 2 0 1 4}$ & $\mathbf{2 0 1 4 / 2 0 1 5}$ \\
\hline December & 7.63 & 8.75 & 21.44 & 23.25 & 14.54 & 16.00 \\
\hline January & 6.35 & 5.52 & 22.42 & 20.48 & 14.39 & 13.00 \\
\hline February & 7.50 & 7.93 & 23.89 & 22.75 & 15.70 & 15.34 \\
\hline March & 11.41 & 12.23 & 26.73 & 27.16 & 19.07 & 19.69 \\
\hline April & 15.83 & 14.59 & 32.80 & 29.10 & 24.32 & 21.84 \\
\hline May & 19.81 & 19.71 & 35.48 & 35.48 & 27.65 & 27.60 \\
\hline June & 22.55 & 21.37 & 37.72 & 36.60 & 30.14 & 28.98 \\
\hline
\end{tabular}

http://eg.freemeteo.com/weather/?language=english \& country = Egypt.

\section{Results}

Phenotypic evaluation

All measured phenotypic traits exhibited highly significant differences and continuous phenotypic variations among evaluated accessions, sowing dates and their interaction in both growing seasons (Table 4) which is expected for quantitative traits.

\section{Plant height $(\mathrm{PH})$}

Plant height $(\mathrm{PH})$ was measured as the height of main stem at maturity. Heat stress conditions led to a significant reduction in plant height as revealed by ANOVA (Table 4). The genotype Assiut 108 revealed the tallest plants under the favorable cultivation conditions (FSD) in both growing seasons (95.89 and 95.33 $\mathrm{cm}$, in the first and second growing seasons, respectively). Under moderately heat-stressed conditions (MHSD), line 1 showed the lowest reduction in plant height $(2.55 \%$ and $0.21 \%)$ in the $1^{s t}$ and $2^{\text {nd }}$ seasons, respectively, as compared with the normal conditions. On the other hand, the highest plants recorded by cultivars Giza 165 in the first growing season $(82.22 \mathrm{~cm})$ and Sakha 8 in the second season $(88.00 \mathrm{~cm})$. Under severely heat-stressed conditions (SHSD) line 1457 and cultivar Gemmeza 7 showed the lowest reduction on (PH) (15.96\% and $4.09 \%$ ) in the first and the second seasons, respectively, as compared with normal conditions, while Giza168 and Gemmeza 7 exhibited the tallest plants in the first and the second seasons, respectively (Table 5). 
TABLE 3. Average of stress tolerance index (STI) of all evaluated traits under severe stress conditions in the two growing seasons.

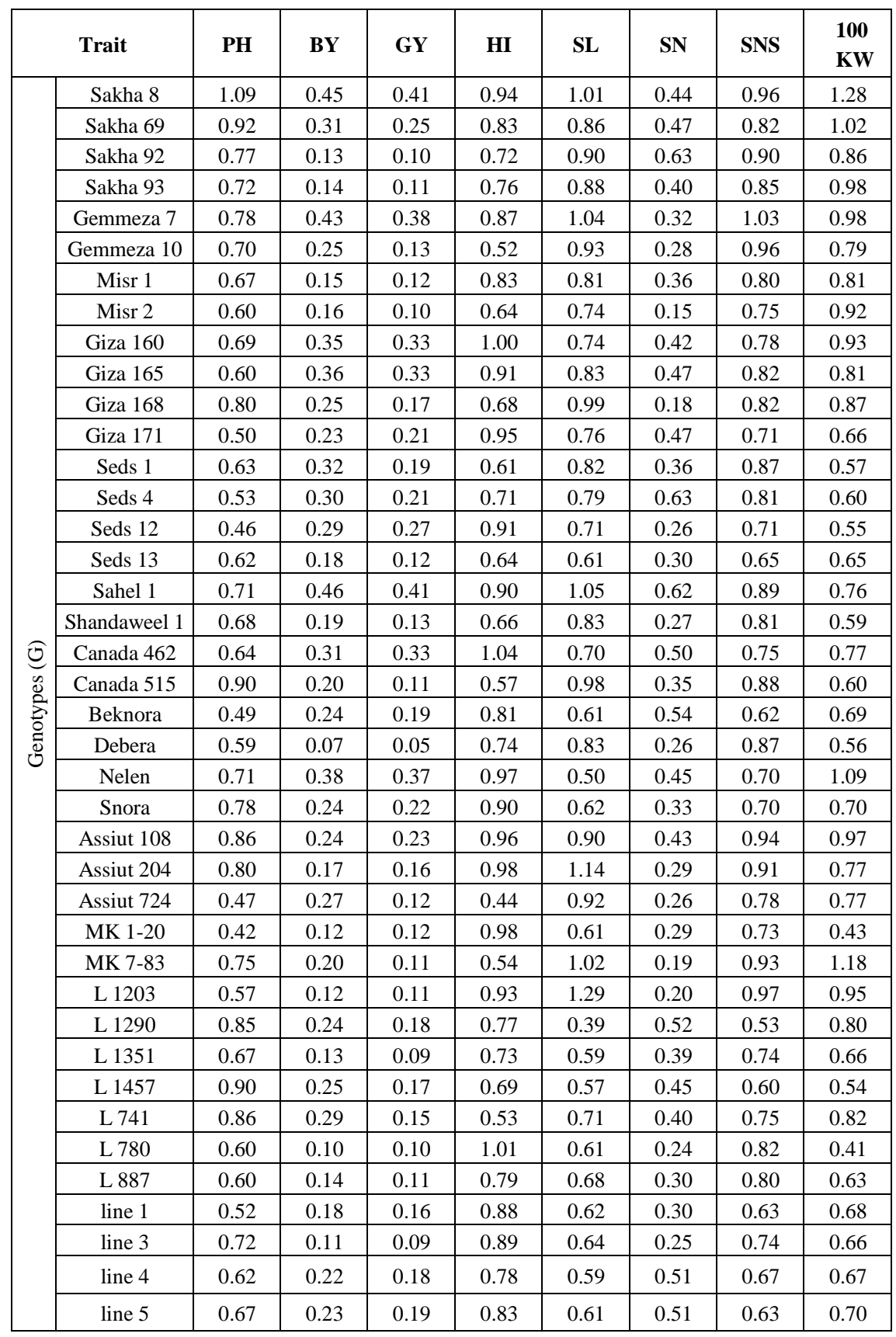

Egypt. J. Agron. 38, No. 3 (2016) 
HEAT STRESS IMPACT AND GENETIC DIVERSITY AMONG ...

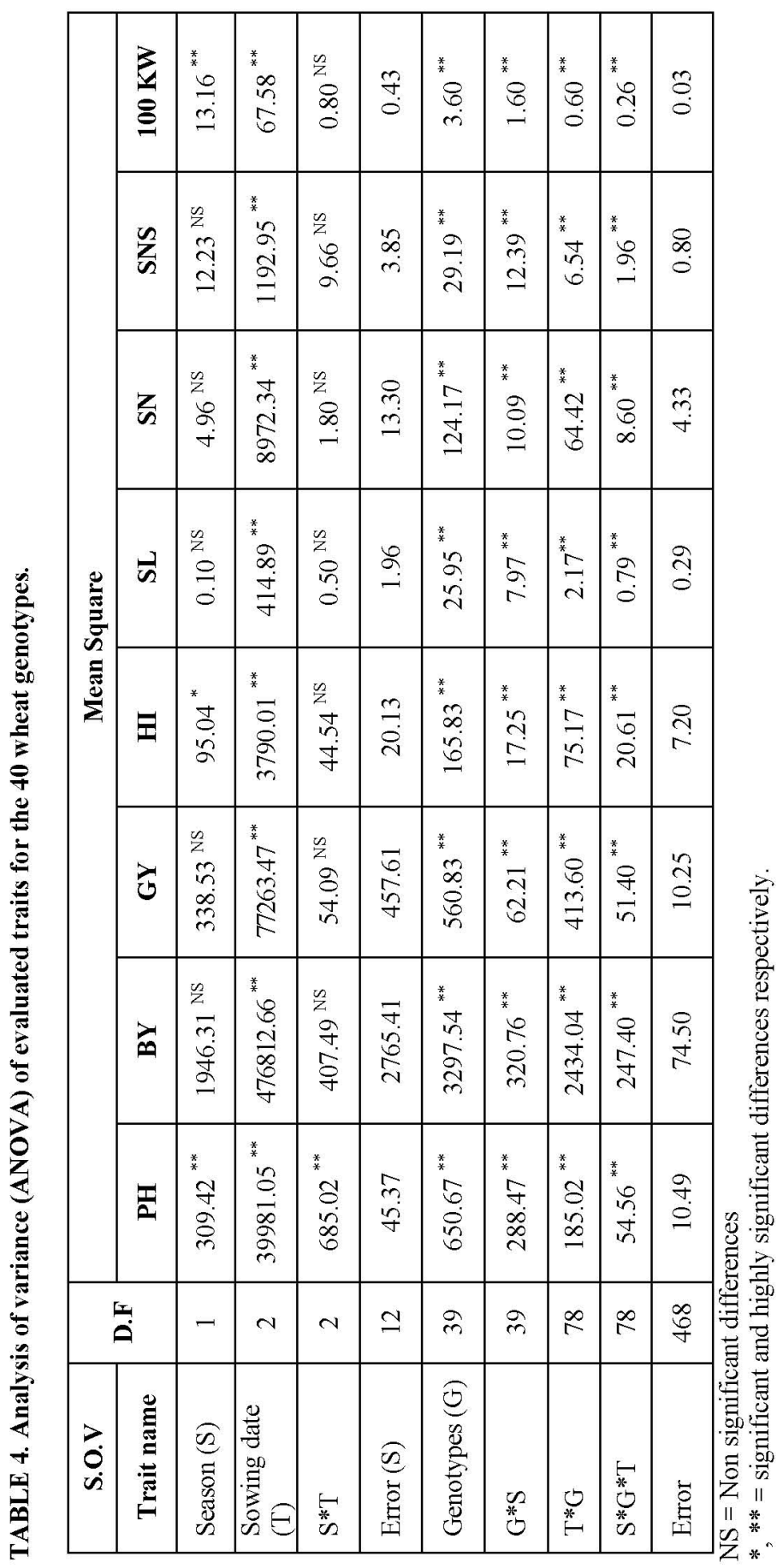

Egypt. J. Agron. 38, No.3 (2016) 
TABLE 5. Plant height (PH) mean performance.

\begin{tabular}{|c|c|c|c|c|c|c|c|c|c|c|c|c|c|}
\hline \multirow{2}{*}{\multicolumn{2}{|c|}{$\begin{array}{c}\text { Season }(\mathbf{S}) \\
\text { Sowing } \\
\text { Date }(\mathbf{T})\end{array}$}} & \multicolumn{4}{|c|}{$2013 / 2014$} & \multicolumn{4}{|c|}{$2014 / 2015$} & \multicolumn{3}{|c|}{ Over all Mean } & \multirow{2}{*}{$\sum_{0}^{\mathbb{E}}$} \\
\hline & & \multirow{2}{*}{$\begin{array}{l}\text { 星 } \\
90.44\end{array}$} & \multirow{2}{*}{$\frac{0}{\sum_{2}^{2}}$} & \multirow{2}{*}{$\begin{array}{c}\text { 昰 } \\
65.89\end{array}$} & \multirow{2}{*}{$\frac{\underset{\Xi}{\Xi}}{77.78}$} & \multirow{2}{*}{$\frac{2}{95}$} & \multirow{2}{*}{$\frac{\text { क्ञ }}{88.00}$} & \multirow{2}{*}{$\begin{array}{c}\text { 官 } \\
67.67\end{array}$} & \multirow{2}{*}{$\frac{\underset{\overparen{E}}{\Sigma}}{83.00}$} & \multirow{2}{*}{$\frac{\text { 公 }}{91.89}$} & \multirow{2}{*}{ 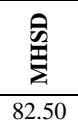 } & \multirow{2}{*}{ 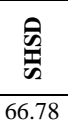 } & \\
\hline \multirow{40}{*}{ 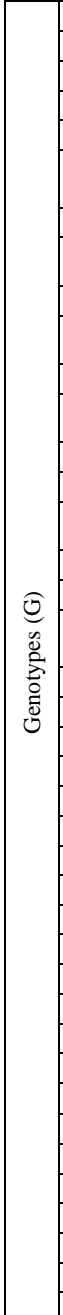 } & Sakha 8 & & & & & & & & & & & & 80.39 \\
\hline & Sakha 69 & 83.89 & 68.56 & 63.33 & 71.93 & 92.33 & 87.17 & 70.83 & 83.44 & 88.11 & 77.86 & 67.08 & 77.69 \\
\hline & Sakha 92 & 69.00 & 54.56 & 47.22 & 56.93 & 91.00 & 84.67 & 73.33 & 83.00 & 80.00 & 69.61 & 60.28 & 69.96 \\
\hline & Sakha 93 & 67.22 & 52.22 & 39.89 & 53.11 & 89.83 & 81.33 & 73.33 & 81.50 & 78.53 & 66.78 & 56.61 & 67.31 \\
\hline & Gemmeza 7 & 93.22 & 74.11 & 46.67 & 71.33 & 77.33 & 76.83 & 74.17 & 76.11 & 85.28 & 75.47 & 60.42 & 73.72 \\
\hline & \begin{tabular}{|c|} 
Gemmeza \\
10
\end{tabular} & 92.89 & 75.33 & 52.22 & 73.48 & 80.17 & 69.33 & 53.17 & 67.56 & 86.53 & 72.33 & 52.69 & 70.52 \\
\hline & Misr 1 & 80.00 & 60.56 & 52.78 & 64.44 & 78.33 & 65.67 & 57.00 & 67.00 & 79.17 & 63.11 & 54.89 & 65.72 \\
\hline & Misr 2 & 83.00 & 78.56 & 39.56 & 67.04 & 75.33 & 68.00 & 58.83 & 67.39 & 79.17 & 73.28 & 49.19 & 67.21 \\
\hline & Giza 160 & 94.11 & 67.56 & 52.67 & 71.44 & 80.33 & 66.83 & 50.33 & 65.83 & 87.22 & 67.19 & 51.50 & 68.64 \\
\hline & Giza 165 & 88.89 & 82.22 & 50.00 & 73.70 & 72.83 & 63.28 & 46.00 & 60.70 & 80.86 & 72.75 & 48.00 & 67.20 \\
\hline & Giza 168 & 87.56 & 80.78 & 72.22 & 80.19 & 76.67 & 67.00 & 53.50 & 65.72 & 82.11 & 73.89 & 62.86 & 72.95 \\
\hline & Giza 171 & 78.22 & 76.11 & 36.33 & 63.56 & 69.67 & 63.28 & 51.33 & 61.43 & 73.94 & 69.69 & 43.83 & 62.49 \\
\hline & Seds 1 & 80.44 & 70.44 & 54.11 & 68.33 & 80.17 & 64.00 & 47.67 & 63.94 & 80.31 & 67.22 & 50.89 & 66.14 \\
\hline & Seds 4 & 75.00 & 57.78 & 50.11 & & 62.56 & 59.00 & 50.11 & & 68.78 & 58.39 & 50.11 & 59.09 \\
\hline & Seds 12 & 83.22 & 65.56 & 44.44 & 64.41 & 55.11 & 52.33 & 42.78 & 50.07 & 69.17 & 58.94 & 43.61 & 57.24 \\
\hline & Seds 13 & 90.44 & 71.78 & 46.67 & 69.63 & 90.17 & 70.67 & 42.22 & 67.69 & 90.31 & 71.22 & 44.44 & 68.66 \\
\hline & Sahel 1 & 91.89 & 60.89 & 50.00 & 67.59 & 93.83 & 60.33 & 49.83 & 68.00 & 92.86 & 60.61 & 49.92 & 67.80 \\
\hline & $\begin{array}{c}\text { Shandaweel } \\
1\end{array}$ & 76.33 & 60.44 & 57.56 & 64.78 & 75.50 & 63.33 & 58.33 & 65.72 & 75.92 & 61.89 & 57.94 & 65.25 \\
\hline & Canada 462 & 78.56 & 73.22 & 47.56 & 66.44 & 76.33 & 74.33 & 59.00 & 69.89 & 77.44 & 73.78 & 53.28 & 68.17 \\
\hline & Canada 515 & 89.11 & 70.44 & 64.11 & 74.56 & 89.67 & 70.17 & 66.78 & 75.54 & 89.39 & 70.31 & 65.44 & 75.05 \\
\hline & Beknora & 62.78 & 61.11 & 50.44 & 58.11 & 64.17 & 61.67 & 48.89 & 58.24 & 63.47 & 61.39 & 49.67 & 58.18 \\
\hline & Debera & 75.00 & 70.89 & 51.67 & 65.85 & 75.00 & 70.33 & 50.83 & 65.39 & 75.00 & 70.61 & 51.25 & 65.62 \\
\hline & Nelen & 87.56 & 63.44 & 46.67 & 65.89 & 87.83 & 64.17 & 57.83 & 69.94 & 87.69 & 63.81 & 52.25 & 67.92 \\
\hline & Snora & 80.00 & 69.00 & 60.56 & 69.85 & 80.00 & 70.33 & 65.44 & 71.93 & 80.00 & 69.67 & 63.00 & 70.89 \\
\hline & Assiut 108 & 95.89 & 65.33 & 61.11 & 74.11 & 95.33 & 74.50 & 55.83 & 75.22 & 95.61 & 69.92 & 58.47 & 74.67 \\
\hline & Assiut 204 & 84.22 & 81.33 & 57.78 & 74.44 & 85.33 & 80.50 & 64.67 & 76.83 & 84.78 & 80.92 & 61.22 & 75.64 \\
\hline & Assiut 724 & 74.00 & 70.89 & 42.22 & 62.37 & 71.50 & 70.33 & 42.50 & 61.44 & 72.75 & 70.61 & 42.36 & 61.91 \\
\hline & MK 1-20 & 62.00 & 58.44 & 40.44 & 53.63 & 62.00 & 58.17 & 47.78 & 55.98 & 62.00 & 58.31 & 44.11 & 54.81 \\
\hline & MK 7-83 & 79.56 & 75.11 & 57.22 & 70.63 & 79.83 & 75.67 & 64.78 & 73.43 & 79.69 & 75.39 & 61.00 & 72.03 \\
\hline & L 1203 & 71.33 & 55.44 & 51.33 & 59.37 & 70.50 & 57.67 & 52.11 & 60.09 & 70.92 & 56.56 & 51.72 & 59.73 \\
\hline & L 1290 & 87.22 & 67.22 & 62.33 & 72.26 & 85.83 & 68.89 & 65.00 & 73.24 & 86.53 & 68.06 & 63.67 & 72.75 \\
\hline & L 1351 & $\frac{01.22}{76.44}$ & 67.89 & $\begin{array}{l}02.50 \\
55.11\end{array}$ & 66.48 & $\frac{00.05}{76.17}$ & 67.33 & 58.22 & 67.24 & $\frac{00.53}{76.31}$ & 67.61 & $\frac{03.01}{56.67}$ & 66.86 \\
\hline & L 1457 & 83.56 & 76.56 & 70.22 & 76.78 & 83.83 & 79.83 & 69.33 & 77.67 & 83.69 & 78.19 & 69.78 & 77.22 \\
\hline & L 741 & 92.11 & 71.56 & 62.22 & 75.30 & 92.67 & 71.83 & 58.44 & 74.31 & 92.39 & 71.69 & 60.33 & 74.81 \\
\hline & L 780 & 76.44 & 72.22 & 47.78 & 65.48 & 76.17 & 70.83 & 55.00 & 67.33 & 76.31 & 71.53 & 51.39 & 66.41 \\
\hline & L 887 & 80.44 & 59.56 & 46.33 & 62.11 & 80.17 & 59.83 & 51.28 & 63.76 & 80.31 & 59.69 & 48.81 & 62.94 \\
\hline & line 1 & 82.67 & 80.56 & 40.44 & 67.89 & 81.00 & 80.83 & 42.44 & 68.09 & 81.83 & 80.69 & 41.44 & 67.99 \\
\hline & line 3 & 78.56 & 67.56 & 55.89 & & 78.83 & 71.83 & 63.17 & & 78.69 & 69.69 & 59.53 & 69.31 \\
\hline & line 4 & 71.11 & 66.56 & 52.89 & 63.52 & 71.67 & 66.83 & 59.17 & 65.89 & 71.39 & 66.69 & 56.03 & $\begin{array}{l}64.70 \\
\end{array}$ \\
\hline & line 5 & 86.67 & 61.56 & 49.56 & 65.93 & 87.50 & 61.83 & 50.67 & 66.67 & 87.08 & 61.69 & 50.11 & 66.30 \\
\hline \multicolumn{2}{|c|}{ Mean } & 81.52 & 68.51 & 52.39 & 67.47 & 79.65 & 69.47 & 56.74 & 68.62 & 80.59 & 68.99 & 54.56 & 68.05 \\
\hline \multicolumn{2}{|c|}{ LSD' $_{0.05}(\mathrm{~S})$} & 1.10 & & & & & LSD' & (G) & & 1.86 & & & \\
\hline LSD & $0.05(\mathrm{~T})$ & 1.21 & & & & & LSD' & $\left(G^{*}\right.$ & & 2.63 & & & \\
\hline LSD & ${ }_{0.05}(\mathrm{~S} * \mathrm{~T})$ & 1.80 & & & & & LSD' & $5\left(U^{*}\right.$ & & 3.32 & & & \\
\hline & & & & & & & LSD' & $05\left(G^{*} S\right.$ & & 5.10 & & & \\
\hline
\end{tabular}

Egypt. J. Agron. 38, No. 3 (2016) 


\section{Biological yield per plant $(B Y)$}

Selection for Biological yield may be useful trait for yield improvement in wheat (Sharma, 1993). In this study (BY) was severely decreased under heatstressed conditions (Table 6). Under the favorable sowing, superiority was scored by Gemmeza 7 and Nelen in the first and second growing seasons, respectively. Meanwhile, under MHSD minimum reduction in BY was recorded by line Assiut 204 (22.3\%) in both growing seasons as compared with normal conditions. Finally, under SHSD, Assiut 204 recorded the lowest reduction as compared with FSD on (BY) in the $1^{\text {st }}$ season, whereas in the $2^{\text {nd }}$ season line 887 was scored the lowest reduction (Table 6).

\section{Grain yield/ plant (GY)}

Grain yield is a complex trait that highly influenced by many genetic and environmental factors (Liu et al., 2008). Grain yield (GY) was significantly decreased under heat-stressed conditions as compared by the normal sowing conditions, (Table 3 and 7). Under (FSD) Gemmeza 7 and Nelen yielded the highest grain yield/ plant $(80.88$ and $87.21 \mathrm{gm} /$ plant, in the first and second growing seasons, respectively). Under MHSD grain yield was decreased with all genotypes in this study, cultivar Sahel 1 yielded the highest grain yield/ plant (17.77) in the $1^{s t}$ season, while in the second season cultivar Seds 4 recorded the highest yield $(22.85 \mathrm{gm} /$ plant). Under SHSD, the lowest reduction in GY was recorded by line Assiut 204 in the $1^{\text {st }}$ season and cultivar line 887 in the second season, moreover superiority in GY under sever condition was recorded by cultivar Beknora (10.24 gm/ plant) in the first season and Giza 160 (14.42 gm/ plant) in the second season (Table 7).

\section{Harvest index (HI)}

Harvest index was measured as the ratio of economic yield to biological yield following the method of Donald \& Hamblin (1976). Harvest index was highly significantly influenced by genotypes, sowing dates and their interaction (Table 3). In normal sowing conditions Giza 171 and Canada 462 exhibited the highest HI (46.51 and 46.99) in the first and second growing seasons, respectively. On the other hand, Debera gave the best performance in HI $\left((40.47 \%)\right.$ under MHSD conditions in the $1^{\text {st }}$ season, but in the $2^{\text {nd }}$ season the superiority was to Giza 168 (42.49\%). Under SHSD conditions, L780 and cultivar Giza165 exhibited the highest HI (41.34 and 37.01) in the first and second growing seasons, respectively (Suppl. Table 8), while the maximum reduction in HI was recorded by cultivar Gemmeza 10 in both growing seasons. 
TABLE 6. Mean performance of biological yield per plant (BY).

\begin{tabular}{|c|c|c|c|c|c|c|c|c|c|c|c|c|c|}
\hline \multirow{2}{*}{\multicolumn{2}{|c|}{$\begin{array}{c}\text { Season }(\mathbf{S}) \\
\text { Sowing } \\
\text { Date }(\mathbf{T})\end{array}$}} & \multicolumn{4}{|c|}{$2013 / 2014$} & \multicolumn{4}{|c|}{$2014 / 2015$} & \multicolumn{3}{|c|}{ Over all Mean } & \multirow{2}{*}{ 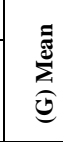 } \\
\hline & & 空 & 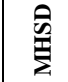 & 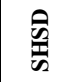 & 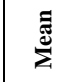 & ชิ & 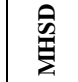 & 卷 & 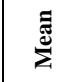 & 空 & 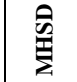 & 产 & \\
\hline \multirow{40}{*}{$\begin{array}{l}\widehat{0} \\
0 \\
0 \\
0 \\
0 \\
0 \\
0 \\
0\end{array}$} & Sakha 8 & 179.65 & 40.40 & 29.55 & 83.20 & 179.65 & 41.51 & 29.55 & \begin{tabular}{|l|}
83.57 \\
\end{tabular} & 179.65 & 40.96 & 29.55 & 83.39 \\
\hline & Sakha 69 & 81.81 & 39.27 & 32.80 & \begin{tabular}{|l|}
51.29 \\
\end{tabular} & 131.62 & 42.18 & 34.49 & \begin{tabular}{|l|}
69.43 \\
\end{tabular} & 106.71 & 40.72 & 33.65 & 60.36 \\
\hline & Sakha 92 & 82.60 & 32.27 & 19.55 & 44.81 & 77.10 & 45.78 & 19.55 & 47.48 & 79.85 & 39.03 & 19.55 & 46.14 \\
\hline & \begin{tabular}{|l|} 
Sakha 93 \\
\end{tabular} & 68.70 & 29.27 & 18.10 & \begin{tabular}{|l|}
38.69 \\
\end{tabular} & \begin{tabular}{|l|}
71.95 \\
\end{tabular} & 47.25 & 27.63 & \begin{tabular}{|l|}
48.94 \\
\end{tabular} & 70.33 & 38.26 & \begin{tabular}{|l|}
22.87 \\
\end{tabular} & 43.82 \\
\hline & Gemmeza 7 & 206.63 & 31.63 & 26.77 & 88.34 & 181.55 & 32.68 & 25.41 & 79.88 & 194.09 & 32.16 & 26.09 & 84.11 \\
\hline & \begin{tabular}{|c|} 
Gemmeza \\
10 \\
\end{tabular} & 118.20 & 32.17 & 26.71 & 59.03 & 108.20 & 38.00 & 24.77 & 56.99 & 113.20 & 35.08 & 25.74 & 58.01 \\
\hline & Misr 1 & 64.41 & 37.26 & 25.37 & \begin{tabular}{|l|l|}
42.34 \\
\end{tabular} & \begin{tabular}{|l}
78.22 \\
\end{tabular} & 35.38 & 24.28 & \begin{tabular}{|l|l|}
45.96 \\
\end{tabular} & 71.31 & 36.32 & 24.83 & 44.15 \\
\hline & Misr 2 & 73.13 & 35.80 & 29.40 & 46.11 & 69.29 & 52.80 & 23.35 & \begin{tabular}{|l|}
48.48 \\
\end{tabular} & 71.21 & 44.30 & 26.38 & 47.29 \\
\hline & Giza 160 & 105.17 & 59.80 & 26.07 & 63.68 & 118.50 & 63.89 & 45.47 & 75.95 & 111.83 & 61.85 & 35.77 & 69.82 \\
\hline & Giza 165 & 135.63 & 33.18 & 31.03 & 66.61 & 140.43 & 49.21 & 30.62 & \begin{tabular}{|l|}
73.42 \\
\end{tabular} & 138.03 & 41.20 & 30.83 & 70.02 \\
\hline & Giza 168 & 123.84 & 33.53 & 26.50 & 61.29 & 112.82 & 38.45 & 23.12 & 58.13 & 118.33 & 35.99 & 24.81 & 59.71 \\
\hline & Giza 171 & 130.53 & 28.80 & 18.67 & 59.33 & 116.45 & 35.35 & 24.44 & $\mathbf{5 8 . 7 5}$ & 123.49 & 32.08 & 21.55 & 59.04 \\
\hline & Seds 1 & 111.30 & 35.32 & 30.13 & 58.92 & 114.18 & 42.88 & 35.84 & 64.30 & 112.74 & 39.10 & 32.99 & 61.61 \\
\hline & Seds 4 & 103.00 & 37.99 & 32.56 & $\mathbf{5 7 . 8 5}$ & 103.63 & 66.12 & 35.98 & 68.58 & 103.31 & 52.05 & 34.27 & 63.21 \\
\hline & Seds 12 & 108.75 & 38.00 & 32.17 & \begin{tabular}{|l|}
59.64 \\
\end{tabular} & 108.75 & 40.92 & 31.20 & 60.29 & 108.75 & 39.46 & \begin{tabular}{|l|}
31.68 \\
\end{tabular} & 59.96 \\
\hline & Seds 13 & 99.38 & 33.77 & 24.80 & 52.65 & \begin{tabular}{|l|}
77.27 \\
\end{tabular} & 29.25 & 22.02 & 42.84 & 88.32 & 31.51 & 23.41 & 47.75 \\
\hline & \begin{tabular}{|l|} 
Sahel 1 \\
\end{tabular} & 181.06 & 52.59 & 30.21 & 87.95 & 195.08 & 54.53 & 27.17 & 92.26 & 188.07 & 53.56 & \begin{tabular}{|l|}
28.69 \\
\end{tabular} & 90.11 \\
\hline & $\begin{array}{c}\text { Shandaweel } \\
1\end{array}$ & 91.93 & 31.78 & 23.07 & 48.93 & 87.35 & 39.70 & 27.87 & 51.64 & 89.64 & 35.74 & 25.47 & 50.28 \\
\hline & Canada 462 & 122.23 & 28.59 & 24.38 & \begin{tabular}{|l|}
58.40 \\
\end{tabular} & 143.40 & 48.20 & 30.40 & \begin{tabular}{|l|}
74.00 \\
\end{tabular} & 132.82 & 38.39 & 27.39 & 66.20 \\
\hline & Canada 515 & 95.53 & 34.94 & 26.93 & \begin{tabular}{|l|}
52.47 \\
\end{tabular} & \begin{tabular}{|l|}
71.20 \\
\end{tabular} & 35.17 & 28.12 & 44.83 & 83.37 & 35.06 & \begin{tabular}{|l|}
27.53 \\
\end{tabular} & 48.65 \\
\hline & Beknora & 93.84 & 44.73 & 32.70 & 57.09 & 99.07 & 31.05 & 24.52 & 51.54 & 96.46 & 37.89 & 28.61 & 54.32 \\
\hline & Debera & 46.65 & 29.93 & 17.76 & \begin{tabular}{|l|}
31.45 \\
\end{tabular} & \begin{tabular}{|l|}
46.65 \\
\end{tabular} & 27.13 & \begin{tabular}{|l|}
19.62 \\
\end{tabular} & \begin{tabular}{|l|}
31.13 \\
\end{tabular} & 46.65 & 28.53 & \begin{tabular}{|l|}
18.69 \\
\end{tabular} & 31.29 \\
\hline & Nelen & 143.54 & 35.73 & 25.87 & \begin{tabular}{|l|}
68.38 \\
\end{tabular} & 203.27 & 46.54 & 25.39 & 91.73 & 173.41 & 41.14 & \begin{tabular}{|l|}
25.63 \\
\end{tabular} & 80.06 \\
\hline & \begin{tabular}{|c|} 
Snora \\
\end{tabular} & 159.49 & 34.24 & 21.80 & 71.84 & 115.18 & 29.47 & 18.19 & 54.28 & 137.34 & 31.86 & \begin{tabular}{|l|}
20.00 \\
\end{tabular} & 63.06 \\
\hline & \begin{tabular}{|l|} 
Assiut 108 \\
\end{tabular} & 115.32 & 45.37 & 21.93 & \begin{tabular}{|l|}
60.87 \\
\end{tabular} & 146.43 & 30.35 & 21.40 & 66.06 & 130.88 & 37.86 & 21.67 & 63.47 \\
\hline & \begin{tabular}{|l|} 
Assiut 204 \\
\end{tabular} & 61.69 & 47.92 & 30.46 & \begin{tabular}{|l|}
46.69 \\
\end{tabular} & 79.48 & 61.73 & 26.48 & 55.90 & 70.59 & 54.83 & 28.47 & 51.29 \\
\hline & \begin{tabular}{|l|} 
Assiut 724 \\
\end{tabular} & 117.97 & 36.73 & 20.10 & 58.27 & 124.68 & 44.03 & 31.58 & 66.76 & 121.32 & 40.38 & 25.84 & 62.51 \\
\hline & \begin{tabular}{|l|} 
MK 1-20 \\
\end{tabular} & 76.07 & 26.93 & 13.34 & \begin{tabular}{|l|}
38.78 \\
\end{tabular} & 72.65 & 31.94 & 25.78 & 43.46 & 74.36 & 29.44 & \begin{tabular}{|l|}
19.56 \\
\end{tabular} & 41.12 \\
\hline & \begin{tabular}{|l|} 
MK 7-83 \\
\end{tabular} & 100.84 & 32.47 & 19.67 & \begin{tabular}{|l|}
50.99 \\
\end{tabular} & 126.32 & 60.93 & 21.05 & 69.43 & 113.58 & 46.70 & 20.36 & 60.21 \\
\hline & L 1203 & 73.20 & 23.10 & 19.19 & 38.50 & 63.45 & 32.03 & 20.78 & 38.76 & 68.33 & 27.57 & 19.99 & 38.63 \\
\hline & L 1290 & 96.93 & 39.57 & 26.99 & 54.50 & 125.85 & 59.21 & 23.43 & 69.50 & 111.39 & 49.39 & 25.21 & 62.00 \\
\hline & L 1351 & 80.10 & 34.00 & 16.57 & 43.56 & 101.35 & 32.46 & \begin{tabular}{|l|}
17.75 \\
\end{tabular} & \begin{tabular}{|l|}
50.52 \\
\end{tabular} & 90.73 & 33.23 & \begin{tabular}{|l|}
17.16 \\
\end{tabular} & 47.04 \\
\hline & L 1457 & 141.35 & 33.87 & 21.51 & 65.58 & 141.35 & 53.88 & 20.57 & 71.93 & 141.35 & 43.88 & 21.04 & 68.75 \\
\hline & L 741 & 157.67 & 36.34 & 19.28 & \begin{tabular}{|l|}
71.10 \\
\end{tabular} & 147.75 & 43.57 & 24.93 & 72.08 & 152.71 & 39.96 & 22.11 & 71.59 \\
\hline & L 780 & 68.24 & 26.11 & 15.40 & 36.59 & 73.22 & 27.09 & 18.40 & 39.57 & 70.73 & 26.60 & 16.90 & 38.08 \\
\hline & L 887 & 62.10 & 33.35 & 23.53 & \begin{tabular}{|l|}
39.66 \\
\end{tabular} & \begin{tabular}{|l|}
62.10 \\
\end{tabular} & 34.17 & 29.76 & 42.01 & 62.10 & 33.76 & \begin{tabular}{|l|}
26.65 \\
\end{tabular} & 40.83 \\
\hline & line 1 & 94.27 & 31.01 & 22.60 & \begin{tabular}{|l|}
49.29 \\
\end{tabular} & \begin{tabular}{|l|}
95.60 \\
\end{tabular} & 29.63 & 22.02 & 49.08 & 94.93 & 30.32 & 22.31 & 49.19 \\
\hline & line 3 & 74.08 & 28.38 & 21.47 & 41.31 & \begin{tabular}{|l|}
65.07 \\
\end{tabular} & 45.02 & 15.82 & 41.97 & 69.57 & 36.70 & \begin{tabular}{|l|}
18.64 \\
\end{tabular} & 41.64 \\
\hline & line 4 & 115.91 & 37.13 & 25.97 & \begin{tabular}{|l|}
59.67 \\
\end{tabular} & \begin{tabular}{|l|}
76.22 \\
\end{tabular} & 38.96 & 28.90 & 48.03 & 96.06 & 38.05 & 27.43 & 53.85 \\
\hline & $\begin{array}{ll}\text { line } 5 \\
\end{array}$ & 122.22 & 43.23 & 21.28 & 62.24 & 118.08 & 38.95 & 22.85 & 59.96 & 120.15 & 41.09 & 22.06 & 61.10 \\
\hline \multicolumn{2}{|c|}{ Mean } & 107.12 & 35.66 & 24.30 & 55.70 & 109.26 & 41.93 & 25.76 & 58.99 & 108.19 & 38.80 & 25.03 & 57.34 \\
\hline \multicolumn{2}{|c|}{ LSD' $_{0.05}(\mathrm{~S})$} & NS & & & & \multicolumn{4}{|c|}{ LSD' $_{0.05}(\mathrm{G})$} & \multicolumn{3}{|l|}{4.95} & \\
\hline \multicolumn{2}{|c|}{ LSD' $_{0.05}(\mathrm{~T})$} & 9.46 & & & & \multicolumn{4}{|c|}{ LSD' $_{0.05}(\mathrm{G} * \mathrm{~S})$} & 8.10 & & & \\
\hline \multirow{2}{*}{\multicolumn{2}{|c|}{ LSD' $_{0.05}\left(\mathrm{~S}^{*} \mathrm{~T}\right)$}} & NS & & & & \multicolumn{4}{|c|}{ LSD' $_{0.05}\left(\mathrm{G}^{*} \mathrm{~T}\right)$} & 8.57 & & & \\
\hline & & & & & & \multicolumn{4}{|c|}{$\mathrm{LSD}^{\prime}{ }_{0.05}\left(\mathrm{G}^{*} \mathrm{~S} * \mathrm{~T}\right)$} & 14.47 & & & \\
\hline
\end{tabular}

Egypt. J. Agron. 38, No. 3 (2016) 
TABLE 7. Mean performance of grain yield per plant (GY).

\begin{tabular}{|c|c|c|c|c|c|c|c|c|c|c|c|c|c|}
\hline \multirow{2}{*}{\multicolumn{2}{|c|}{\begin{tabular}{|c|} 
Season $(\mathbf{S})$ \\
Sowing \\
Date $(\mathrm{T})$
\end{tabular}}} & \multicolumn{4}{|c|}{$2013 / 2014$} & \multicolumn{4}{|c|}{$2014 / 2015$} & \multicolumn{3}{|c|}{ Over all Mean } & \multirow{2}{*}{ 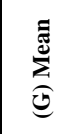 } \\
\hline & & \%ิ & $\stackrel{0}{\stackrel{2}{E}}$ & $\stackrel{0}{\tilde{0}}$ & 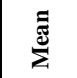 & \%ิ & 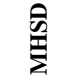 & 卷 & 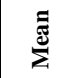 & \%ิ & 党 & 卷 & \\
\hline \multirow{40}{*}{$\begin{array}{l}0 \\
0 \\
0 \\
0 \\
0 \\
0 \\
0 \\
0 \\
0\end{array}$} & Sakha 8 & 63.90 & 12.23 & 10.03 & 28.72 & 67.95 & 13.35 & 10.80 & 30.70 & 65.93 & 12.79 & 10.42 & 29.71 \\
\hline & Sakha 69 & 30.80 & 12.76 & 10.00 & 17.85 & 53.17 & 14.32 & 10.25 & 25.91 & 41.98 & 13.54 & 10.13 & 21.88 \\
\hline & Sakha 92 & 34.47 & 8.28 & 4.90 & 15.88 & 31.32 & 15.79 & 4.90 & 17.34 & 32.90 & 12.03 & 4.90 & 16.61 \\
\hline & Sakha 93 & 20.32 & 11.41 & 5.53 & 12.42 & 26.29 & 15.63 & 9.50 & 17.14 & 23.31 & 13.52 & 7.52 & 14.78 \\
\hline & Gemmeza 7 & 80.88 & 10.79 & 8.51 & 33.40 & 69.84 & 11.43 & 8.22 & 29.83 & 75.36 & 11.11 & 8.37 & 31.61 \\
\hline & $\begin{array}{c}\text { Gemmeza } \\
10\end{array}$ & 42.00 & 6.47 & 4.83 & 17.77 & 40.06 & 7.67 & 5.55 & 17.76 & 41.03 & 7.07 & 5.19 & 17.77 \\
\hline & Misr 1 & 24.73 & 11.30 & 7.35 & 14.46 & 28.50 & 10.38 & 8.16 & 15.68 & 26.61 & 10.84 & 7.75 & 15.07 \\
\hline & Misr 2 & 20.50 & 13.17 & 7.62 & 13.76 & 22.83 & 15.50 & 7.92 & 15.42 & 21.67 & 14.33 & 7.77 & 14.59 \\
\hline & Giza 160 & 43.86 & 16.14 & 9.13 & 23.04 & 47.63 & 19.83 & 14.42 & 27.29 & 45.75 & 17.99 & 11.78 & 25.17 \\
\hline & Giza 165 & 54.67 & 11.34 & 8.13 & 24.71 & 57.63 & 17.86 & 11.35 & 28.95 & 56.15 & 14.60 & 9.74 & 26.83 \\
\hline & Giza 168 & 39.31 & 13.37 & 7.47 & 20.05 & 38.37 & 16.33 & 7.01 & 20.57 & 38.84 & 14.85 & 7.24 & 20.31 \\
\hline & Giza 171 & 60.87 & 10.21 & 5.29 & 25.46 & 49.39 & 13.22 & 7.84 & 23.48 & 55.13 & 11.71 & 6.57 & 24.47 \\
\hline & Seds 1 & 39.98 & 10.86 & 7.14 & \begin{tabular}{|l|}
19.33 \\
\end{tabular} & 40.36 & 11.93 & 8.85 & 20.38 & 40.17 & 11.40 & 8.00 & 19.86 \\
\hline & Seds 4 & 34.04 & 12.64 & 10.17 & 18.95 & 30.96 & 22.85 & 11.57 & 21.80 & 32.50 & 17.75 & 10.87 & 20.37 \\
\hline & Seds 12 & 42.45 & 13.38 & 9.60 & 21.81 & 42.45 & 13.24 & 11.34 & 22.34 & 42.45 & 13.31 & 10.47 & 22.08 \\
\hline & Seds 13 & 35.36 & 11.68 & 6.93 & \begin{tabular}{|l|}
17.99 \\
\end{tabular} & 26.07 & 9.31 & 5.53 & 13.64 & 30.72 & 10.49 & 6.23 & 15.81 \\
\hline & \begin{tabular}{|c|} 
Sahel 1 \\
\end{tabular} & 77.12 & 17.77 & 9.48 & 34.79 & 77.34 & 20.59 & 8.16 & 35.36 & 77.23 & 19.18 & 8.82 & 35.08 \\
\hline & $\begin{array}{c}\text { Shandaweel } \\
1\end{array}$ & 32.55 & 9.15 & 6.12 & 15.94 & 28.39 & 10.36 & 7.83 & 15.53 & 30.47 & 9.76 & 6.98 & 15.74 \\
\hline & Canada 462 & 55.99 & 9.66 & 7.67 & 24.44 & 67.43 & 17.35 & 9.84 & 31.54 & 61.71 & 13.51 & 8.75 & 27.99 \\
\hline & Canada 515 & 32.08 & 9.53 & 6.44 & 16.02 & 23.86 & 8.72 & 6.87 & 13.15 & 27.97 & 9.13 & 6.65 & 14.58 \\
\hline & \begin{tabular}{|l|} 
Beknora \\
\end{tabular} & 33.90 & 16.21 & 10.24 & 20.12 & 35.65 & 11.07 & 7.84 & 18.19 & 34.77 & 13.64 & 9.04 & 19.15 \\
\hline & Debera & 18.38 & 12.10 & 4.33 & 11.61 & 18.30 & 10.04 & 5.64 & 11.33 & 18.34 & 11.07 & 4.99 & 11.47 \\
\hline & Nelen & 55.90 & 13.70 & 8.36 & 25.99 & 87.21 & 17.17 & 8.95 & 37.78 & 71.56 & 15.44 & 8.65 & 31.88 \\
\hline & Snora & 64.36 & 13.50 & 7.44 & 28.43 & 46.48 & 11.15 & 5.40 & 21.01 & 55.42 & 12.33 & 6.42 & 24.72 \\
\hline & Assiut 108 & 46.29 & 13.76 & 7.57 & 22.54 & 59.86 & 9.21 & 7.11 & 25.39 & 53.08 & 11.48 & 7.34 & 23.97 \\
\hline & Assiut 204 & 25.09 & 13.50 & 10.16 & 16.25 & 31.63 & 19.90 & 9.23 & 20.25 & 28.36 & 16.70 & 9.69 & 18.25 \\
\hline & Assiut 724 & 34.00 & 13.63 & 4.03 & 17.22 & 40.50 & 15.39 & 6.50 & 20.80 & 37.25 & 14.51 & 5.27 & 19.01 \\
\hline & MK 1-20 & 34.16 & 9.60 & 4.14 & 15.97 & 31.29 & 10.12 & 8.38 & 16.60 & 32.73 & 9.86 & 6.26 & 16.28 \\
\hline & MK 7-83 & 32.97 & 9.90 & 3.82 & 15.57 & 39.43 & 20.47 & 5.84 & 21.92 & 36.20 & 15.19 & 4.83 & 18.74 \\
\hline & L 1203 & 31.87 & 7.57 & 5.90 & 15.11 & 25.56 & 8.72 & 6.71 & 13.67 & 28.72 & 8.15 & 6.30 & 14.39 \\
\hline & L 1290 & 35.05 & 12.29 & 8.62 & 18.65 & 45.53 & 20.26 & 6.57 & 24.12 & 40.29 & 16.28 & 7.59 & 21.39 \\
\hline & L 1351 & 30.24 & 8.71 & 4.48 & 14.48 & 38.40 & 8.59 & 4.71 & 17.23 & 34.32 & 8.65 & 4.60 & 15.85 \\
\hline & L 1457 & 41.55 & 8.73 & 7.31 & 19.20 & 41.55 & 22.32 & 6.49 & 23.45 & 41.55 & 15.52 & 6.90 & 21.33 \\
\hline & L 741 & 49.03 & 7.60 & 5.01 & 20.55 & 45.08 & 10.16 & 5.81 & 20.35 & 47.05 & 8.88 & 5.41 & 20.45 \\
\hline & L 780 & 26.09 & 8.09 & 6.30 & \begin{tabular}{|l|}
13.49 \\
\end{tabular} & 25.97 & 10.02 & 6.55 & 14.18 & 26.03 & 9.06 & 6.43 & 13.84 \\
\hline & L 887 & 22.25 & 10.40 & 7.12 & 13.26 & 22.25 & 11.06 & 9.71 & 14.34 & 22.25 & 10.73 & 8.42 & 13.80 \\
\hline & line 1 & 34.28 & 11.10 & 7.28 & 17.55 & 37.73 & 9.36 & 7.23 & 18.11 & 36.00 & 10.23 & 7.26 & 17.83 \\
\hline & line 3 & 30.99 & 8.55 & 5.03 & 14.86 & 26.88 & 18.29 & 5.80 & 16.99 & 28.94 & 13.42 & 5.42 & 15.92 \\
\hline & line 4 & 53.61 & 12.81 & 6.70 & 24.38 & 31.79 & 14.25 & 7.69 & 17.91 & 42.70 & 13.53 & 7.19 & 21.14 \\
\hline & line 5 & 48.47 & 13.65 & 6.05 & 22.72 & 48.30 & 13.05 & 7.18 & 22.84 & 48.38 & 13.35 & 6.61 & 22.78 \\
\hline \multicolumn{2}{|c|}{ Mean } & 40.36 & 11.44 & 7.06 & 19.62 & 41.23 & 13.91 & 7.88 & 21.01 & 40.80 & 12.67 & 7.47 & 20.31 \\
\hline \multicolumn{2}{|c|}{$\mathrm{LSD}_{0.05}(\mathrm{~S})$} & NS & & & & \multicolumn{4}{|c|}{ LSD' $_{0.05}(\mathrm{G})$} & \multicolumn{3}{|l|}{1.84} & \\
\hline \multicolumn{2}{|c|}{ LSD' $_{0.05}(\mathrm{~T})$} & 3.85 & & & & \multicolumn{4}{|c|}{ LSD' $_{0.05}\left(\mathrm{G}^{*} \mathrm{~S}\right)$} & 2.87 & & & \\
\hline \multirow{2}{*}{\multicolumn{2}{|c|}{ LSD' $_{0.05}\left(\mathrm{~S}^{*} \mathrm{~T}\right)$}} & NS & & & & \multicolumn{4}{|c|}{ LSD' $_{0.05}\left(\mathrm{G}^{*} \mathrm{~T}\right)$} & 3.18 & & & \\
\hline & & & & & & \multicolumn{4}{|c|}{$\mathrm{LSD}^{\prime}{ }_{0.05}(\mathrm{G} * \mathrm{~S} * \mathrm{~T})$} & 5.06 & & & \\
\hline
\end{tabular}


TABLE 8. Mean performance of harvest index per plant (HI).

\begin{tabular}{|c|c|c|c|c|c|c|c|c|c|c|c|c|c|}
\hline \multirow{2}{*}{\multicolumn{2}{|c|}{$\begin{array}{c}\text { Season }(\mathbf{S}) \\
\text { Sowing } \\
\text { Date }(\mathbf{T})\end{array}$}} & \multicolumn{4}{|c|}{$2013 / 2014$} & \multicolumn{4}{|c|}{$2014 / 2015$} & \multicolumn{3}{|c|}{ Over all Mean } & \multirow{2}{*}{$\sum_{0}^{\mathbb{E}}$} \\
\hline & & \multirow{2}{*}{ क्ञ } & \multirow{2}{*}{ 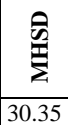 } & \multirow{2}{*}{\begin{tabular}{|l}
$\frac{2}{\sqrt[5]{\sigma}}$ \\
34.39
\end{tabular}} & \multirow{2}{*}{ 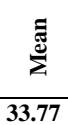 } & \multirow{2}{*}{$\begin{array}{l}\text { के } \\
37.95\end{array}$} & \multirow{2}{*}{${ }_{32.51}^{\text {\% }}$} & \multirow{2}{*}{ 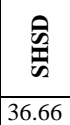 } & \multirow{2}{*}{ 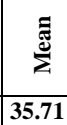 } & \multirow{2}{*}{$\begin{array}{r}\text { \% } \\
37.27\end{array}$} & \multirow{2}{*}{$\sum_{31.43}^{\text {क्र }}$} & \multirow{2}{*}{$\begin{array}{l}\text { क्ञ } \\
\frac{\pi}{5} \\
35.53\end{array}$} & \\
\hline \multirow{40}{*}{ 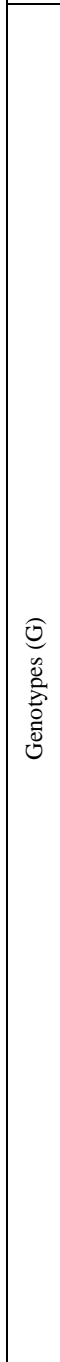 } & Sakha 8 & & & & & & & & & & & & 34.74 \\
\hline & Sakha 69 & 37.31 & 32.44 & 30.56 & 33.44 & 40.30 & 33.84 & 29.73 & 34.63 & 38.81 & 33.14 & 30.14 & 34.03 \\
\hline & Sakha 92 & 41.86 & 25.74 & 24.73 & 30.78 & 40.63 & 33.56 & 24.98 & 33.06 & 41.25 & 29.65 & 24.85 & 31.92 \\
\hline & Sakha 93 & 29.57 & 38.95 & 30.47 & 33.00 & 36.34 & 34.15 & 34.35 & 34.95 & 32.95 & 36.55 & 32.41 & 33.97 \\
\hline & Gemmeza 7 & 39.13 & 34.02 & 31.65 & 34.93 & 38.45 & 34.89 & 32.21 & 35.18 & 38.79 & 34.46 & 31.93 & 35.06 \\
\hline & $\begin{array}{c}\text { Gemmeza } \\
10\end{array}$ & 35.57 & 20.18 & 18.06 & 24.60 & 37.06 & 20.10 & 22.39 & 26.51 & 36.32 & 20.14 & 20.22 & 25.56 \\
\hline & Misr 1 & 38.32 & 30.34 & 29.05 & 32.57 & 36.47 & 29.22 & 33.61 & 33.10 & 37.39 & 29.78 & 31.33 & 32.83 \\
\hline & Misr 2 & 28.00 & 36.77 & 25.77 & \begin{tabular}{|l|}
30.18 \\
\end{tabular} & 32.88 & 30.52 & 33.25 & 32.22 & 30.44 & 33.64 & 29.51 & 31.20 \\
\hline & Giza 160 & 41.70 & 27.34 & 34.99 & 34.68 & 40.28 & 30.92 & 32.43 & $\mathbf{3 4 . 5 4}$ & 40.99 & 29.13 & 33.71 & 34.61 \\
\hline & Giza 165 & 40.30 & 34.20 & 26.19 & 33.56 & 41.03 & 37.00 & 37.01 & 38.35 & 40.66 & 35.60 & 31.60 & 35.96 \\
\hline & Giza 168 & 31.86 & 39.79 & 28.19 & 33.28 & 34.10 & 42.49 & 30.24 & 35.61 & 32.98 & 41.14 & 29.22 & 34.45 \\
\hline & Giza 171 & 46.51 & 35.36 & 28.73 & 36.87 & 42.28 & 37.37 & 31.67 & 37.11 & 44.40 & 36.36 & 30.20 & 36.99 \\
\hline & Seds 1 & 35.93 & 30.74 & 23.52 & 30.06 & 35.35 & 27.76 & 24.66 & 29.26 & 35.64 & 29.25 & 24.09 & 29.66 \\
\hline & Seds 4 & 33.05 & 33.27 & 31.45 & 32.59 & 29.88 & 34.45 & 32.18 & 32.17 & 31.46 & 33.86 & 31.81 & 32.38 \\
\hline & Seds 12 & 39.00 & 35.21 & 29.64 & 34.62 & 39.02 & 32.62 & 36.40 & 36.02 & 39.01 & 33.91 & 33.02 & 35.32 \\
\hline & Seds 13 & 35.39 & 34.83 & 27.92 & 32.71 & 33.53 & 31.80 & 24.79 & 30.04 & 34.46 & 33.31 & 26.35 & 31.38 \\
\hline & Sahel 1 & 43.06 & 33.76 & 31.46 & 36.10 & 39.74 & 37.74 & 29.98 & 35.82 & 41.40 & 35.75 & 30.72 & 35.96 \\
\hline & $\begin{array}{c}\text { Shandaweel } \\
1 \\
\end{array}$ & 35.37 & 28.51 & 26.67 & 30.18 & 32.47 & 26.12 & 28.31 & 28.97 & 33.92 & 27.31 & 27.49 & 29.57 \\
\hline & Canada 462 & 45.78 & 33.71 & 31.50 & 36.99 & 46.99 & 36.20 & 31.78 & 38.32 & 46.38 & 34.95 & 31.64 & 37.66 \\
\hline & Canada 515 & 33.58 & 27.30 & 23.76 & 28.21 & 33.50 & 24.81 & 24.38 & 27.56 & 33.54 & 26.05 & 24.07 & 27.89 \\
\hline & Beknora & 36.10 & 36.09 & 31.53 & 34.57 & 35.98 & 35.47 & 31.99 & 34.48 & 36.04 & 35.78 & 31.76 & 34.53 \\
\hline & Debera & 39.59 & 40.47 & 24.42 & $\mathbf{3 4 . 8 3}$ & 39.28 & 36.94 & 28.42 & 34.88 & 39.44 & 38.71 & 26.42 & 34.85 \\
\hline & Nelen & 38.73 & 38.22 & 32.14 & 36.36 & 42.77 & 37.00 & 35.25 & 38.34 & 40.75 & 37.61 & 33.69 & 37.35 \\
\hline & Snora & 40.09 & 38.73 & 34.08 & 37.63 & 40.25 & 37.63 & 29.71 & 35.86 & 40.17 & 38.18 & 31.90 & 36.75 \\
\hline & \begin{tabular}{|l|} 
Assiut 108 \\
\end{tabular} & 40.14 & 30.81 & 32.89 & 34.62 & 40.86 & 30.33 & 34.06 & 35.08 & 40.50 & 30.57 & 33.47 & 34.85 \\
\hline & \begin{tabular}{|l} 
Assiut 204 \\
\end{tabular} & 40.23 & 28.14 & 34.20 & 34.19 & 39.89 & 31.74 & 34.79 & \begin{tabular}{|l|}
35.47 \\
\end{tabular} & 40.06 & 29.94 & 34.50 & 34.83 \\
\hline & Assiut 724 & 28.75 & 37.27 & 20.51 & 28.84 & 32.44 & 35.03 & 20.48 & 29.32 & 30.59 & 36.15 & 20.49 & 29.08 \\
\hline & \begin{tabular}{|l|} 
MK 1-20 \\
\end{tabular} & 44.55 & 35.94 & 30.63 & 37.04 & 42.95 & 31.64 & 32.59 & 35.73 & 43.75 & 33.79 & 31.61 & 36.38 \\
\hline & MK 7-83 & 32.71 & 30.53 & 19.63 & 27.62 & 31.25 & 32.34 & 28.26 & 30.62 & 31.98 & 31.43 & 23.95 & 29.12 \\
\hline & L 1203 & 43.43 & 32.82 & 30.62 & 35.62 & 40.15 & 27.23 & 32.29 & 33.22 & 41.79 & 30.03 & 31.45 & 34.42 \\
\hline & L 1290 & 36.16 & 30.51 & 31.82 & 32.83 & 36.18 & 33.28 & 28.08 & 32.51 & 36.17 & 31.89 & 29.95 & 32.67 \\
\hline & L 1351 & 37.71 & 25.94 & 27.74 & 30.46 & 37.88 & 26.41 & 26.57 & 30.29 & 37.79 & 26.18 & 27.16 & 30.37 \\
\hline & L 1457 & 29.79 & 25.96 & 33.99 & 29.91 & 29.49 & 40.47 & 31.47 & 33.81 & 29.64 & 33.22 & 32.73 & 31.86 \\
\hline & L 741 & 31.09 & 20.84 & 25.81 & 25.91 & 30.50 & 23.27 & 23.29 & 25.68 & 30.79 & 22.05 & 24.55 & 25.80 \\
\hline & L 780 & 38.36 & 31.03 & \begin{tabular}{|l|}
41.34 \\
\end{tabular} & 36.91 & \begin{tabular}{|l}
55.52 \\
\end{tabular} & 37.01 & 35.77 & \begin{tabular}{|l|}
36.10 \\
\end{tabular} & 36.94 & 34.02 & 38.56 & 36.51 \\
\hline & L 887 & 35.70 & \begin{tabular}{|l|}
31.32 \\
\end{tabular} & 30.16 & 32.39 & 35.80 & 32.42 & 32.67 & 33.63 & 35.75 & 31.87 & 31.41 & 33.01 \\
\hline & line 1 & 36.34 & 35.37 & 32.31 & 34.67 & 39.45 & 31.49 & 33.55 & 34.83 & 37.90 & 33.43 & 32.93 & 34.75 \\
\hline & line 3 & 41.90 & 30.07 & 23.61 & 31.86 & 41.32 & 39.88 & 37.00 & 39.40 & 41.61 & 34.97 & 30.30 & 35.63 \\
\hline & line 4 & 46.12 & 34.51 & 25.57 & 35.40 & 40.87 & 36.62 & 25.00 & 34.16 & 43.49 & 35.57 & 25.29 & 34.78 \\
\hline & line 5 & 40.02 & 31.32 & 27.61 & 32.98 & \begin{tabular}{|l|}
41.01 \\
\end{tabular} & 33.47 & 30.56 & 35.01 & 40.51 & 32.40 & 29.08 & 34.00 \\
\hline \multicolumn{2}{|c|}{ Mean } & 37.63 & 32.22 & 28.98 & 32.94 & 37.55 & 32.94 & 30.57 & \begin{tabular}{|l|}
33.69 \\
\end{tabular} & 37.59 & 32.58 & 29.78 & 33.32 \\
\hline \multicolumn{2}{|c|}{ LSD' $_{0.05}(\mathrm{~S})$} & 0.75 & & & & & LSD' 0 & & & 1.58 & & & \\
\hline LSD' & ${ }_{0.05}(\mathrm{~T})$ & 0.81 & & & & & LSD' $_{0}$ & 5 ( 5 ( S) & & 2.87 & & & \\
\hline LSD' & ${ }_{0.05}(\mathrm{~S} * \mathrm{~T})$ & NS & & & & & LSD' $_{0}$ & ${ }_{5}\left(\mathrm{G}^{*} \mathrm{~T}\right)$ & & 2.81 & & & \\
\hline & & & & & & & LSD' $_{0}$ & $G$ & & 4.61 & & & \\
\hline
\end{tabular}

Egypt. J. Agron. 38, No. 3 (2016) 
Spike length (SL)

Grain yield is influenced by spike properties and spike length (Martincic et al., 1996). Spike length (SL) was measured as the length from neck node to the tip of spike at maturity. Elhani et al. (2007) reported that, spike length mainly affected by the growing environment. In our study heat stress conditions led to a significant reduction in spike length. The overall average of spike length was reduced from 11.56 under FSD conditions to 10.07 and $8.94 \mathrm{~cm}$ under MHSD and SHSD conditions, respectively (Table 3 and 9). Under normal conditions cultivars Giza 168 and Sakha 92 recorded the longest spike (15.00 and 15.25 $\mathrm{cm}$ in the first and second growing seasons, respectively). Moreover, Giza 168 was superior in spike length $(14.33 \mathrm{~cm})$ in the $1^{\text {st }}$ season and Sakha $93(12.83$ $\mathrm{cm})$ in the $2^{\text {nd }}$ season under MHSD conditions. Meanwhile, under SHSD conditions L 1203 produced the longest spike $(12.50 \mathrm{~cm})$ in the first growing season and Assiut 204 surpassed all wheat genotypes $(11.75 \mathrm{~cm})$ in the second growing season (Table 9).

\section{Number of spikes/ plant (SN)}

Number of spikes per plant (SN) was significantly influenced by genotypes, sowing date and their interaction (Table 3). Heat-stressed conditions led to a significant reduction in spike number (Table 10). Under FSD conditions the highest SN was observed with cultivar Sahel 1 on both growing seasons, while the lowest number of spikes (11.50 and 10 spikes/ plant) produced by L887 and Misr 2 (in the first and second growing seasons, respectively. Under MHSD conditions line Assiut 724 produced the lowest number of spikes (5.67 spikes/ plant) in the $1^{\text {st }}$ season and cultivar Giza 168 (6.50 spikes/ plant) in the $2^{\text {nd }}$ season, while Line 1290 produced the highest number of spikes (16.33 and 17.50 spikes/ plant, in the first and second growing seasons, respectively). Under conditions of SHSD, cultivars Giza 168 and Misr 2 produced the lowest number of spikes/ plant (4.33 and 4.58, in the first and second growing seasons, respectively). Meanwhile, cultivar Sakha 92 produced the highest number of spikes/ plant (12.50 in both growing seasons) (Table 10).

\section{Spikelet number per spike (SNS)}

There is interdependence and correlation between spike length and spikelet number per spike and spikelet number per spike plays a very important role in the possible increase of grain yield of new genotypes (Martincic et al., 1996). In this study as an average over all genotypes spikelet number per spike (SNS) was significantly reduced from 20.97 to 18.85 and 16.51 due to MHSD and SHSD stress conditions, respectively (Table 11). The highest SNS was recorded by cultivar Gemmeza 10 (25 spikelet per spike) in the $1^{\text {st }}$ season, while Line Assiut 108 (25 spikelet per spike) surpassed all studied genotypes under this study in the $2^{\text {nd }}$ season under normal conditions. Meanwhile, under MHSD conditions Giza 171 and Sakha 8 was superior (22.11 and 22.33) in the $1^{s t}$ and $2^{\text {nd }}$ growing seasons, respectively. Finally, under SHSD, superiority was recorded to Seds 1 (19.67) in the first growing season and Sakha 8 (20.67) in the $2^{\text {nd }}$ growing season (Table 11). 
TABLE 9. Mean performance of spike length (SL).

\begin{tabular}{|c|c|c|c|c|c|c|c|c|c|c|c|c|c|}
\hline \multirow{2}{*}{\multicolumn{2}{|c|}{$\begin{array}{c}\text { Season (S) } \\
\text { Sowing } \\
\text { Date }(\mathbf{T})\end{array}$}} & \multicolumn{4}{|c|}{$2013 / 2014$} & \multicolumn{4}{|c|}{$2014 / 2015$} & \multicolumn{3}{|c|}{ Over all Mean } & \multirow{3}{*}{ 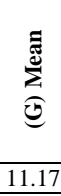 } \\
\hline & & \multirow{2}{*}{ 产 } & \multirow{2}{*}{$\sum_{10.17}^{\text {क्ष }}$} & \multirow{2}{*}{$\frac{2}{5}$} & \multirow{2}{*}{$\sum_{\Sigma}^{\Xi \Xi}$} & \multirow{2}{*}{$\begin{array}{l}\text { \% } \\
13.25 \\
\end{array}$} & \multirow{2}{*}{\begin{tabular}{|l}
$\stackrel{\text { क }}{E}$ \\
12.25 \\
\end{tabular}} & \multirow{2}{*}{$\begin{array}{l}\frac{2}{5} \\
9.33\end{array}$} & \multirow{2}{*}{ 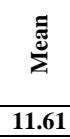 } & \multirow{2}{*}{$\frac{\text { 公 }}{12.68}$} & \multirow{2}{*}{$\sum_{11.21}^{\stackrel{2}{2}}$} & \multirow{2}{*}{ 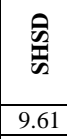 } & \\
\hline \multirow{40}{*}{$\begin{array}{l}00 \\
0 \\
0 \\
0 \\
0 \\
0 \\
0 \\
0 \\
0\end{array}$} & Sakha 8 & & & & & & & & & & & & \\
\hline & Sakha 69 & 10.61 & 10.00 & 8.39 & 9.67 & 14.33 & 11.67 & 9.83 & 11.94 & 12.47 & 10.83 & 9.11 & 10.81 \\
\hline & $\begin{array}{l}\text { Sakha } 92 \\
\end{array}$ & 10.28 & 9.11 & 8.17 & 9.19 & 15.25 & 12.75 & 10.17 & 12.72 & 12.76 & 10.93 & 9.17 & 10.95 \\
\hline & \begin{tabular}{|l|} 
Sakha 93 \\
\end{tabular} & 9.44 & 8.78 & 7.72 & 8.65 & 14.00 & 12.83 & 11.50 & 12.78 & 11.72 & 10.81 & 9.61 & 10.71 \\
\hline & Gemmeza 7 & 13.39 & 11.00 & 10.94 & 11.78 & 12.83 & 11.42 & 10.33 & 11.53 & 13.11 & 11.21 & 10.64 & 11.65 \\
\hline & $\begin{array}{c}\text { Gemmeza } \\
10\end{array}$ & 14.00 & 11.78 & 10.06 & 11.94 & 11.58 & 10.17 & 9.33 & 10.36 & 12.79 & 10.97 & 9.69 & 11.15 \\
\hline & Misr 1 & 11.56 & 9.61 & 8.78 & 9.98 & 11.25 & 10.67 & 10.17 & 10.69 & 11.40 & 10.14 & 9.47 & 10.34 \\
\hline & Misr 2 & 11.50 & 9.61 & 9.11 & 10.07 & 10.92 & 9.75 & 8.50 & 9.72 & 11.21 & 9.68 & 8.81 & 9.90 \\
\hline & Giza 160 & 11.06 & 10.44 & 8.78 & 10.09 & 12.00 & 9.83 & 8.42 & 10.08 & 11.53 & 10.14 & 8.60 & 10.09 \\
\hline & Giza 165 & 12.61 & 11.50 & 9.28 & 11.13 & 11.92 & 9.92 & 8.72 & 10.19 & 12.26 & 10.71 & 9.00 & 10.66 \\
\hline & Giza 168 & 15.00 & 14.33 & 10.67 & 13.33 & 12.00 & 10.33 & 8.67 & 10.33 & 13.50 & 12.33 & 9.67 & 11.83 \\
\hline & Giza 171 & 12.78 & 12.11 & 7.44 & 10.78 & 11.50 & 10.17 & 9.33 & 10.33 & 12.14 & 11.14 & 8.39 & 10.56 \\
\hline & Seds 1 & 14.17 & 10.61 & 9.72 & 11.50 & 11.00 & 8.55 & 7.33 & 8.96 & 12.58 & 9.58 & 8.53 & 10.23 \\
\hline & Seds 4 & 11.67 & 10.83 & 10.28 & 10.93 & 10.64 & 9.58 & 8.60 & 9.61 & 11.15 & 10.21 & 9.44 & 10.27 \\
\hline & Seds 12 & 13.00 & 11.89 & 8.83 & 11.24 & 10.00 & 9.00 & 7.56 & 8.85 & 11.50 & 10.44 & 8.19 & 10.05 \\
\hline & Seds 13 & 10.00 & 9.33 & 8.56 & 9.30 & 10.00 & 9.25 & 7.75 & 9.00 & 10.00 & 9.29 & 8.15 & 9.15 \\
\hline & \begin{tabular}{|l|} 
Sahel 1 \\
\end{tabular} & 13.50 & 10.89 & 10.56 & 11.65 & 13.50 & 10.33 & 10.31 & 11.38 & 13.50 & 10.61 & 10.43 & 11.51 \\
\hline & $\begin{array}{c}\text { Shandaweel } \\
1\end{array}$ & 11.50 & 9.78 & 8.67 & 9.98 & 11.50 & 11.17 & 10.50 & 11.06 & 11.50 & 10.47 & 9.58 & 10.52 \\
\hline & Canada 462 & 10.67 & 10.11 & 8.00 & 9.59 & 11.50 & 10.67 & 8.81 & 10.32 & 11.08 & 10.39 & 8.40 & 9.96 \\
\hline & \begin{tabular}{|l|} 
Canada 515 \\
\end{tabular} & 13.83 & 10.22 & 9.56 & 11.20 & 13.00 & 10.14 & 9.92 & 11.02 & 13.42 & 10.18 & 9.74 & 11.11 \\
\hline & Beknora & 10.00 & 8.94 & 8.03 & 8.99 & 9.83 & 8.58 & 8.39 & 8.94 & 9.92 & 8.76 & 8.21 & 8.96 \\
\hline & Debera & 12.11 & 10.00 & 9.22 & 10.44 & 11.42 & 10.33 & 9.75 & 10.50 & 11.76 & 10.17 & 9.49 & 10.47 \\
\hline & Nelen & 10.00 & 6.78 & 6.44 & 7.74 & 10.00 & 8.86 & 6.92 & 8.59 & 10.00 & 7.82 & 6.68 & 8.17 \\
\hline & \begin{tabular}{|l|} 
Snora \\
\end{tabular} & 9.78 & 8.83 & 8.61 & 9.07 & 9.92 & 9.25 & 8.31 & 9.16 & 9.85 & 9.04 & 8.46 & 9.12 \\
\hline & Assiut 108 & 13.67 & 10.33 & 8.94 & 10.98 & 13.25 & 9.50 & 8.83 & 10.53 & 13.46 & 9.92 & 8.89 & 10.75 \\
\hline & \begin{tabular}{|l|} 
Assiut 204 \\
\end{tabular} & 13.89 & 11.83 & 10.67 & 12.13 & 13.33 & 12.25 & 11.75 & 12.44 & 13.61 & 12.04 & 11.21 & 12.29 \\
\hline & Assiut 724 & 14.44 & 10.94 & 8.33 & 11.24 & 14.17 & 10.67 & 8.83 & 11.22 & 14.31 & 10.81 & 8.58 & 11.23 \\
\hline & MK 1-20 & 9.78 & 8.33 & 7.83 & 8.65 & 10.03 & 9.17 & 8.58 & 9.26 & 9.90 & 8.75 & 8.21 & 8.95 \\
\hline & MK 7-83 & 12.89 & 11.39 & 11.11 & 11.80 & 12.33 & 12.08 & 10.50 & 11.64 & 12.61 & 11.74 & 10.81 & 11.72 \\
\hline & L 1203 & 14.89 & 12.89 & 12.50 & 13.43 & 14.33 & 12.33 & 11.17 & 12.61 & 14.61 & 12.61 & 11.83 & 13.02 \\
\hline & L 1290 & 7.67 & 7.00 & 6.67 & 7.11 & 8.08 & 7.17 & 6.58 & 7.28 & 7.88 & 7.08 & 6.63 & 7.19 \\
\hline & L 1351 & 9.33 & 8.89 & 8.28 & 8.83 & 9.75 & 8.97 & 8.33 & 9.02 & 9.54 & 8.93 & 8.31 & 8.93 \\
\hline & L 1457 & 9.22 & 8.33 & 7.94 & 8.50 & 9.75 & 8.83 & 8.08 & 8.89 & 9.49 & 8.58 & 8.01 & 8.69 \\
\hline & L 741 & 10.56 & 9.61 & 9.11 & 9.76 & 10.83 & 9.42 & 8.61 & 9.62 & 10.69 & 9.51 & 8.86 & 9.69 \\
\hline & L 780 & 11.22 & 8.67 & 6.61 & 8.83 & 11.08 & 8.83 & 7.92 & 9.28 & 11.15 & 8.75 & 7.26 & 9.06 \\
\hline & L 887 & 10.56 & 9.22 & 8.67 & 9.48 & 10.83 & 9.08 & 8.33 & 9.42 & 10.69 & 9.15 & 8.50 & 9.45 \\
\hline & line 1 & 10.61 & 10.44 & 7.80 & 9.62 & 11.17 & 10.17 & 7.42 & 9.58 & 10.89 & 10.31 & 7.61 & 9.60 \\
\hline & line 3 & 9.89 & 9.72 & 9.00 & 9.54 & 9.75 & 9.08 & 8.33 & 9.06 & 9.82 & 9.40 & 8.67 & 9.30 \\
\hline & line 4 & 10.33 & 9.17 & 7.89 & 9.13 & 9.50 & 8.75 & 8.08 & 8.78 & 9.92 & 8.96 & 7.99 & 8.95 \\
\hline & line 5 & 10.00 & 9.44 & 8.00 & 9.15 & 10.00 & 9.17 & 8.25 & 9.14 & 10.00 & 9.31 & 8.13 & 9.14 \\
\hline \multicolumn{2}{|c|}{ Mean } & 11.59 & 10.07 & 8.88 & 10.18 & 11.53 & 10.07 & 8.95 & 10.19 & 11.56 & 10.07 & 8.91 & 10.18 \\
\hline \multicolumn{2}{|c|}{ LSD' $_{0.05}(\mathrm{~S})$} & NS & & & & & LSD' $_{0}$ & ${ }_{5}(\mathrm{G})$ & & 0.31 & & & \\
\hline LSD & $0.05(\mathrm{~T})$ & 0.25 & & & & & LSD' $_{0}$ & ${ }_{5}(\mathrm{G} * \mathrm{~S})$ & & 0.44 & & & \\
\hline עה & $0.05(\mathrm{~S} * \mathrm{~T})$ & NS & & & & & LSD' $_{0}$ & ${ }_{5}\left(G^{*} \mathrm{~T}\right)$ & & 0.58 & & & \\
\hline & & & & & & & LSD' $_{0}$ & $\left(\mathrm{G} * \mathrm{~S}^{*}\right.$ & & 0.93 & & & \\
\hline
\end{tabular}

Egypt. J. Agron. 38, No. 3 (2016) 
TABLE 10. Mean performance of spike number per plant (SN).

\begin{tabular}{|c|c|c|c|c|c|c|c|c|c|c|c|c|c|}
\hline \multirow{2}{*}{\multicolumn{2}{|c|}{$\begin{array}{c}\text { Season }(\mathbf{S}) \\
\text { Sowing } \\
\text { Date }(\mathbf{T})\end{array}$}} & \multicolumn{4}{|c|}{$2013 / 2014$} & \multicolumn{4}{|c|}{$2014 / 2015$} & \multicolumn{3}{|c|}{ Over all Mean } & \multirow{2}{*}{ 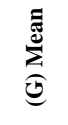 } \\
\hline & & \multirow{2}{*}{$\begin{array}{l}\text { \% } \\
21.30 \\
\end{array}$} & \multirow{2}{*}{ 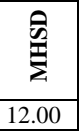 } & \multirow{2}{*}{$\frac{2}{5}$} & \multirow{2}{*}{$\stackrel{\text { Е }}{\text { Е }}$} & \multirow{2}{*}{$\begin{array}{l}\text { \% } \\
23.50 \\
\end{array}$} & \multirow{2}{*}{$\frac{\hat{2}}{\sum^{2}}$} & \multirow{2}{*}{$\begin{array}{l}\text { \% } \\
\text { 芯 } \\
7.50\end{array}$} & \multirow{2}{*}{ 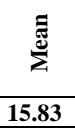 } & \multirow{2}{*}{ 佥 } & \multirow{2}{*}{\begin{tabular}{|c|} 
兽 \\
12.75 \\
\end{tabular}} & \multirow{2}{*}{ 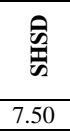 } & \\
\hline \multirow{40}{*}{ 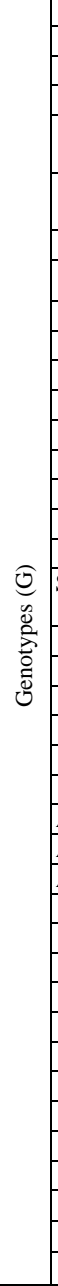 } & Sakha 8 & & & & & & & & & & & & 14.22 \\
\hline & Sakha 69 & 17.67 & 12.67 & 10.00 & 13.44 & 21.00 & 11.83 & 8.75 & 13.86 & 19.34 & 12.25 & 9.38 & 13.66 \\
\hline & Sakha 92 & 19.78 & 14.00 & 12.50 & 15.43 & 18.67 & 15.00 & 12.50 & 15.39 & 19.23 & 14.50 & 12.50 & 15.41 \\
\hline & Sakha 93 & 20.00 & 9.00 & 7.00 & 12.00 & 20.00 & 11.67 & 8.33 & 13.33 & 20.00 & 10.33 & 7.67 & 12.67 \\
\hline & $\begin{array}{c}\text { Gemmeza } \\
7\end{array}$ & 17.67 & 8.33 & 8.00 & 14.67 & 16.00 & 9.17 & 6.25 & 13.81 & 16.84 & 8.75 & 7.13 & 10.91 \\
\hline & $\begin{array}{c}\text { Gemmeza } \\
10\end{array}$ & 17.00 & 8.00 & 7.22 & 10.74 & 15.75 & 8.22 & 5.83 & 9.94 & 16.38 & 8.11 & 6.53 & 10.34 \\
\hline & \begin{tabular}{l|} 
Misr 1 \\
\end{tabular} & \begin{tabular}{|l|}
14.56 \\
\end{tabular} & 12.00 & 9.56 & 12.04 & 16.83 & 11.00 & 8.11 & \begin{tabular}{|l|}
11.98 \\
\end{tabular} & 15.70 & 11.50 & 8.83 & 12.01 \\
\hline & Misr 2 & 11.67 & 10.78 & 6.00 & 9.48 & 10.00 & 9.17 & 4.58 & 7.92 & 10.84 & 9.97 & 5.29 & 8.70 \\
\hline & Giza 160 & 16.67 & 12.25 & 6.67 & 11.86 & 18.75 & 14.46 & 11.38 & 14.86 & 17.71 & 13.35 & 9.02 & 13.36 \\
\hline & Giza 165 & 21.00 & 11.11 & 8.11 & 13.41 & 22.25 & 15.03 & 8.67 & 15.31 & 21.63 & 13.07 & 8.39 & 14.36 \\
\hline & Giza 168 & 15.33 & 6.33 & 4.33 & 8.67 & 14.50 & 6.50 & 4.75 & 8.58 & 14.92 & 6.42 & 4.54 & 8.63 \\
\hline & Giza 171 & 23.67 & 9.44 & 7.67 & 15.26 & 22.00 & 9.78 & 8.17 & 13.31 & 22.84 & 9.61 & 7.92 & 13.46 \\
\hline & Seds 1 & 16.33 & 15.67 & 6.56 & 12.85 & 18.25 & 15.50 & 9.33 & 14.36 & 17.29 & 15.58 & 7.94 & 13.60 \\
\hline & Seds 4 & 22.33 & 11.78 & 8.33 & 14.15 & 27.75 & 14.33 & 10.83 & 17.64 & 25.04 & 13.06 & 9.58 & 15.89 \\
\hline & Seds 12 & 16.50 & 9.33 & 6.33 & 10.72 & 16.50 & 11.83 & 5.50 & \begin{tabular}{|l|}
11.28 \\
\end{tabular} & 16.50 & 10.58 & 5.92 & 11.00 \\
\hline & Seds 13 & 16.78 & 8.67 & 8.00 & 11.15 & 15.67 & 7.67 & 6.00 & 9.78 & 16.23 & 8.17 & 7.00 & 10.47 \\
\hline & \begin{tabular}{l|l} 
Sahel 1 \\
\end{tabular} & 24.44 & 11.89 & 10.33 & 16.22 & 23.67 & 13.83 & 9.39 & 18.96 & 24.06 & 12.86 & 9.86 & 15.59 \\
\hline & $\begin{array}{c}\text { Shandaweel } \\
1\end{array}$ & 17.33 & 8.67 & 6.78 & 10.93 & 14.00 & 8.33 & 6.50 & 9.61 & 15.67 & 8.50 & 6.64 & 10.27 \\
\hline & Canada 462 & \begin{tabular}{|l|}
21.78 \\
\end{tabular} & 9.11 & 7.67 & 12.85 & 23.17 & 11.28 & 9.33 & 14.59 & 22.48 & 10.19 & 8.50 & 13.72 \\
\hline & Canada 515 & 17.00 & 11.78 & 9.33 & 12.70 & 13.25 & 9.50 & 8.33 & 10.36 & 15.13 & 10.64 & 8.83 & 11.53 \\
\hline & Beknora & 23.00 & 14.00 & 9.56 & 15.52 & 20.50 & 11.28 & 9.33 & 13.70 & 21.75 & 12.64 & 9.44 & 14.61 \\
\hline & Debera & 13.00 & 10.11 & 7.89 & 10.33 & 13.00 & 10.17 & 7.17 & 10.11 & 13.00 & 10.14 & 7.53 & 10.22 \\
\hline & Nelen & 20.22 & 11.00 & 8.78 & 15.33 & 22.33 & 10.28 & 7.50 & 16.70 & 21.28 & 10.64 & 8.14 & 13.35 \\
\hline & \begin{tabular}{|l|} 
Snora \\
\end{tabular} & 20.44 & 8.44 & 6.33 & 15.07 & 20.17 & 8.17 & 6.17 & 11.50 & 20.31 & 8.31 & 6.25 & 11.62 \\
\hline & Assiut 108 & 20.78 & 15.56 & 7.67 & 14.67 & 24.67 & 9.00 & 6.75 & 13.47 & 22.73 & 12.28 & 7.21 & 14.07 \\
\hline & Assiut 204 & 12.17 & 11.28 & 8.56 & 10.67 & 13.83 & 11.00 & 8.33 & 11.06 & 13.00 & 11.14 & 8.44 & 10.86 \\
\hline & Assiut 724 & 17.67 & 5.67 & 5.00 & 9.44 & 18.50 & 7.33 & 5.83 & 10.56 & 18.09 & 6.50 & 5.42 & 10.00 \\
\hline & MK 1-20 & 20.78 & 8.33 & 4.56 & 11.22 & 17.17 & 11.33 & 7.50 & 12.00 & 18.98 & 9.83 & 6.03 & 11.61 \\
\hline & MK 7-83 & 13.33 & 8.33 & 5.56 & 9.07 & 15.00 & 11.08 & 4.67 & 10.25 & 14.17 & 9.71 & 5.11 & 9.66 \\
\hline & L 1203 & 14.00 & 5.89 & 5.67 & 8.52 & 11.50 & 9.33 & 6.50 & 9.11 & 12.75 & 7.61 & 6.08 & 8.81 \\
\hline & L 1290 & 18.11 & 16.33 & 10.78 & 15.07 & 23.67 & 17.50 & 8.67 & 16.61 & 20.89 & 16.92 & 9.72 & 15.84 \\
\hline & L 1351 & 17.33 & 10.50 & 8.00 & 11.94 & 20.25 & 9.72 & 8.00 & 12.66 & 18.79 & 10.11 & 8.00 & 12.30 \\
\hline & L 1457 & 20.40 & 14.33 & 9.22 & 21.35 & 21.50 & 12.33 & 7.33 & 20.06 & 20.95 & 13.33 & 8.28 & 14.19 \\
\hline & L 741 & 17.67 & 9.78 & 8.11 & 11.85 & 17.25 & 11.17 & 9.28 & 12.56 & 17.46 & 10.47 & 8.69 & 12.21 \\
\hline & L 780 & 16.22 & 12.11 & 5.33 & 11.22 & 17.33 & 8.58 & 5.75 & 10.56 & 16.78 & 10.35 & 5.54 & 10.89 \\
\hline & L 887 & 11.50 & 10.67 & 9.00 & 10.39 & 13.75 & 11.50 & 9.00 & 11.42 & 12.63 & 11.08 & 9.00 & 10.90 \\
\hline & line 1 & 15.89 & 12.22 & 7.67 & 11.93 & 17.83 & 10.17 & 6.00 & 11.33 & 16.86 & 11.19 & 6.83 & 11.63 \\
\hline & line 3 & 17.56 & 7.33 & 5.67 & 10.19 & 17.83 & 8.58 & 5.00 & 10.47 & 17.70 & 7.96 & 5.33 & 10.33 \\
\hline & line 4 & 23.00 & 10.89 & 9.33 & 14.41 & 18.00 & 12.17 & 9.75 & 13.31 & 20.50 & 11.53 & 9.54 & 13.86 \\
\hline & line 5 & 19.00 & 16.22 & 9.67 & 14.96 & 19.00 & 16.67 & 11.00 & 15.56 & 19.00 & 16.44 & 10.33 & 15.26 \\
\hline \multicolumn{2}{|c|}{ Mean } & 18.02 & 10.80 & 7.76 & 12.63 & 18.36 & 11.12 & 7.74 & 12.84 & 18.19 & 10.96 & 7.75 & 12.30 \\
\hline & LSD' $_{0.05}(\mathrm{~S})$ & \multicolumn{4}{|l|}{ NS } & & & & $0.05(\mathrm{G})$ & 1.19 & & & \\
\hline & LSD' $_{0.05}(\mathrm{~T})$ & 0.66 & & & & & & LSD'。 & 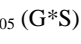 & 2.13 & & & \\
\hline & $\mathrm{SD}^{\prime}{ }_{0.05}\left(\mathrm{~S}^{*} \mathrm{~T}\right)$ & NS & & & & & & LSD' & 格 & 2.15 & & & \\
\hline & & & & & & & & D' ${ }^{\prime} 0$. & $\mathrm{G} * \mathrm{~S} * \mathrm{~T})$ & 3.76 & & & \\
\hline
\end{tabular}


TABLE 11. Spikelet number per spike (SNS) mean performance.

\begin{tabular}{|c|c|c|c|c|c|c|c|c|c|c|c|c|c|}
\hline \multirow{2}{*}{\multicolumn{2}{|c|}{$\begin{array}{c}\text { Season }(\mathbf{S}) \\
\text { Sowing } \\
\text { Date }(\mathbf{T})\end{array}$}} & \multicolumn{4}{|c|}{$2013 / 2014$} & \multicolumn{4}{|c|}{$2014 / 2015$} & \multicolumn{3}{|c|}{ Over all Mean } & \multirow{2}{*}{ 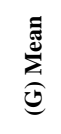 } \\
\hline & & \multirow{2}{*}{ 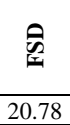 } & \multirow{2}{*}{$\underset{19.00}{\stackrel{0}{E}}$} & \multirow{2}{*}{$\frac{\text { की }}{\text { क }}$} & \multirow{2}{*}{ 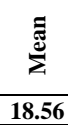 } & \multirow{2}{*}{ 各 } & \multirow{2}{*}{$\frac{\stackrel{0}{\Sigma}}{22.33}$} & \multirow{2}{*}{$\frac{\text { क }}{5}$} & \multirow{2}{*}{$\frac{\text { Еే }}{22.56}$} & \multirow{2}{*}{ 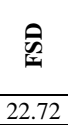 } & \multirow{2}{*}{$\frac{\text { 菖 }}{\Sigma}$} & \multirow{2}{*}{$\frac{\sqrt{0}}{\sqrt{5}}$} & \\
\hline \multirow{40}{*}{$\begin{array}{l}0 \\
0 \\
0 \\
0 \\
0 \\
0 \\
0 \\
0\end{array}$} & Sakha 8 & & & & & & & & & & & & 20.56 \\
\hline & Sakha 69 & 20.00 & 19.00 & 16.78 & 18.59 & 22.00 & 20.67 & 17.67 & 20.11 & 21.00 & 19.83 & 17.22 & 19.35 \\
\hline & Sakha 92 & 20.78 & 17.67 & 15.89 & 18.11 & 24.33 & 21.00 & 19.00 & 21.44 & 22.56 & 19.33 & 17.44 & 19.78 \\
\hline & Sakha 93 & 17.89 & 17.00 & 15.22 & 16.70 & 23.67 & 22.00 & 20.00 & 21.89 & 20.78 & 19.50 & 17.61 & 19.30 \\
\hline & $\begin{array}{c}\text { Gemmeza } \\
7 \\
\end{array}$ & 23.89 & 19.89 & 18.11 & 20.63 & 22.33 & 20.33 & 19.53 & 20.73 & 23.11 & 20.11 & 18.82 & 20.68 \\
\hline & $\begin{array}{c}\text { Gemmeza } \\
10\end{array}$ & 25.00 & 21.00 & 19.00 & 21.67 & 21.67 & 19.33 & 17.00 & 19.33 & 23.33 & 20.17 & 18.00 & 20.50 \\
\hline & Misr 1 & 19.22 & 17.89 & 17.67 & 18.26 & 19.67 & 19.50 & 18.67 & 19.28 & 19.44 & 18.69 & 18.17 & 18.77 \\
\hline & Misr 2 & 21.00 & 19.67 & 15.89 & 18.85 & 20.33 & 18.67 & 15.89 & 18.30 & 20.67 & 19.17 & 15.89 & 18.57 \\
\hline & Giza 160 & 20.89 & 19.22 & 17.00 & 19.04 & 20.67 & 19.17 & 16.17 & 18.67 & 20.78 & 19.19 & 16.58 & 18.85 \\
\hline & Giza 165 & 24.11 & 21.00 & 16.78 & 20.63 & 21.17 & 17.67 & 14.89 & 17.91 & 22.64 & 19.33 & 15.83 & 19.27 \\
\hline & Giza 168 & 22.78 & 21.89 & 17.22 & 20.63 & 21.50 & 19.00 & 15.33 & 18.61 & 22.14 & 20.44 & 16.28 & 19.62 \\
\hline & Giza 171 & 23.00 & 22.11 & 13.22 & 19.44 & 19.78 & 18.67 & 16.17 & 18.20 & 21.39 & 20.39 & 14.69 & 18.82 \\
\hline & Seds 1 & 22.89 & 21.00 & 19.67 & 21.19 & 21.00 & 17.00 & 15.00 & 17.67 & 21.94 & 19.00 & 17.33 & 19.43 \\
\hline & Seds 4 & 1.89 & 18.11 & 17.89 & 19.30 & 19.44 & 18.00 & 16.28 & 17.91 & 20.67 & 18.06 & 17.08 & 8.60 \\
\hline & Seds 12 & 3.00 & 21.00 & 14.11 & 19.37 & 19.00 & 17.33 & 15.56 & 17.30 & 21.00 & 19.17 & 14.83 & 18.33 \\
\hline & Seds 13 & 21.00 & 17.33 & 13.89 & 17.41 & 21.00 & 18.00 & 13.22 & 17.41 & 21.00 & 17.67 & 13.56 & 17.41 \\
\hline & \begin{tabular}{|l|} 
Sahel 1 \\
\end{tabular} & 22.11 & 19.89 & 17.22 & 19.74 & 22.67 & 19.33 & 17.78 & 19.93 & 22.39 & 19.61 & 17.50 & 19.83 \\
\hline & \begin{tabular}{|c|} 
Shandaweel \\
1
\end{tabular} & 21.00 & 16.11 & 16.11 & 17.74 & 21.00 & 19.17 & 17.67 & 19.28 & 21.00 & 17.64 & 16.89 & 18.51 \\
\hline & \begin{tabular}{|l|} 
Canada 462 \\
\end{tabular} & 21.22 & 19.22 & 14.11 & 18.19 & 22.33 & 20.33 & 16.11 & 19.59 & 21.78 & 19.78 & 15.11 & 18.89 \\
\hline & Canada 515 & 23.00 & 19.89 & 16.11 & 19.67 & 23.00 & 19.33 & 17.67 & 20.00 & 23.00 & 19.61 & 16.89 & 19.83 \\
\hline & \begin{tabular}{|l|} 
Beknora \\
\end{tabular} & 19.44 & 17.44 & 13.22 & 16.70 & 18.67 & 17.67 & 15.33 & 7.22 & 19.06 & 17.56 & 14.28 & 16.96 \\
\hline & Debera & 21.89 & 19.00 & 17.22 & 19.37 & 21.33 & 19.00 & 18.00 & 19.44 & 21.61 & 19.00 & 17.61 & 19.41 \\
\hline & Nelen & 21.00 & 15.56 & 13.44 & 16.67 & 21.00 & 17.78 & 15.83 & 18.2 & 21.00 & 16.67 & 14.64 & 17.44 \\
\hline & Snora & 19.00 & 17.00 & 15.78 & 17.26 & 19.00 & 18.17 & 16.61 & 17.93 & 19.00 & 17.58 & 16.19 & 17.59 \\
\hline & Assiut 108 & 25.00 & 17.22 & 15.89 & 19.37 & 25.00 & 17.33 & 17.00 & 19.78 & 25.00 & 17.28 & 16.44 & 19.57 \\
\hline & Assiut 204 & 21.00 & 19.44 & 17.67 & 19.37 & 21.67 & 19.67 & 19.67 & 20.33 & 21.33 & 19.56 & 18.67 & 19.85 \\
\hline & Assiut 724 & 21.89 & 21.00 & 15.22 & 19.37 & 21.33 & 21.00 & 16.33 & 19.56 & 21.61 & 21.00 & 15.78 & 19.46 \\
\hline & MK 1-20 & 20.11 & 18.11 & 15.00 & 17.74 & 20.67 & 18.67 & 16.33 & 18.56 & 20.39 & 18.39 & 15.67 & 18.15 \\
\hline & MK 7-83 & 21.89 & 20.78 & 18.33 & 20.33 & 21.67 & 20.67 & 19.33 & & 21.78 & 20.72 & 18.83 & 20.44 \\
\hline & L 1203 & 23.67 & 20.11 & 18.33 & 20.70 & 22.00 & 20.67 & 19.22 & 20.63 & 22.83 & 20.39 & 18.78 & 20.67 \\
\hline & L 1290 & 15.67 & 15.00 & 15.00 & 15.22 & 16.78 & 15.00 & 14.00 & 15.26 & 16.22 & 15.00 & 14.50 & 15.24 \\
\hline & L 1351 & 19.00 & 18.11 & 17.00 & 18.04 & 19.00 & 18.39 & 17.33 & 18.24 & 19.00 & 18.25 & 17.17 & 18.14 \\
\hline & L 1457 & 16.11 & 15.22 & 15.00 & 15.44 & 18.00 & 16.67 & 15.67 & 16.78 & 17.06 & 15.94 & 15.33 & 16.11 \\
\hline & L 741 & 20.11 & 19.00 & 16.11 & 18.41 & 20.67 & 19.00 & 16.28 & 18.65 & 20.39 & 19.00 & 16.19 & 18.53 \\
\hline & L 780 & 20.78 & 19.44 & 17.00 & 19.07 & 21.00 & 20.00 & 17.67 & 19.56 & 20.89 & 19.72 & 17.33 & 19.31 \\
\hline & L 887 & 21.00 & 17.89 & 17.00 & 18.63 & 21.00 & 17.33 & 16.67 & 18.33 & 21.00 & 17.61 & 16.83 & 18.48 \\
\hline & line 1 & 19.22 & 19.00 & 14.78 & 17.67 & 20.33 & 19.00 & 13.22 & 17.52 & 19.78 & 19.00 & 14.00 & 17.59 \\
\hline & line 3 & 19.00 & 18.11 & 17.67 & 18.26 & 19.00 & 18.67 & 16.67 & 18.11 & 19.00 & 18.39 & 17.17 & 18.19 \\
\hline & line 4 & 20.56 & 17.67 & 14.89 & 17.70 & 18.33 & 16.00 & 15.67 & 16.67 & 19.44 & 16.83 & 15.28 & 17.19 \\
\hline & line 5 & 18.78 & 17.44 & 14.11 & 16.78 & 19.00 & 18.33 & 15.33 & 17.56 & 18.89 & 17.89 & 14.72 & 17.17 \\
\hline \multicolumn{2}{|c|}{ Mean } & 21.01 & 18.76 & 16.16 & 18.65 & 20.92 & 18.90 & 16.81 & 18.88 & 20.97 & 18.83 & 16.49 & 18.76 \\
\hline
\end{tabular}

LSD' $_{0.05}$ (S) NS

LSD' $_{0.05}(\mathrm{~T}) \quad 0.35$

LSD' $_{0.05}\left(\mathrm{~S}^{*} \mathrm{~T}\right) \quad \mathrm{NS}$
LSD' $_{0.05}(\mathrm{G}) \quad 0.51$

LSD' $_{0.05}(\mathrm{G} * \mathrm{~S}) \quad 0.75$

LSD' $_{0.05}\left(\mathrm{G}^{*} \mathrm{~T}\right) \quad 0.95$

LSD' $_{0.05}\left(\mathrm{G}^{*} \mathrm{~S} * \mathrm{~T}\right) \quad 1.57$

Egypt. J. Agron. 38, No. 3 (2016) 


\section{0 kernel weight $(100 \mathrm{KW})$}

It is a well-known fact that 1000- seed weight had a significant effect on yield in cereals (Birol \& Necmettin, 2011). As a general mean of all genotypes the average weight of 100 kernel was significantly reduced from 4.36 under FSD conditions to 3.77 and $3.30 \mathrm{gm}$ under MHSD and SHSD conditions, respectively (Table 12). Under FSD conditions, cultivars Giza 160 and Sakha 8 produced the highest values of $100 \mathrm{KW}$ (5.66 and $5.98 \mathrm{gm}$ ) in the $1^{\text {st }}$ and $2^{\text {nd }}$ growing seasons, respectively. For MHSD conditions cultivars Nelen exhibited the highest values of $100 \mathrm{KW}$ (4.55 and $5.00 \mathrm{gm}$ ) in the first and second season, respectively. Finally, under SHSD conditions, superiority in $100 \mathrm{KW}$ was recorded to Giza 168 (4.02 $\mathrm{gm}$ in the first growing season) and Sakha 92 (4.87 gm in the second growing season) (Table 12).

\section{Cluster analysis}

Cluster analysis using stress tolerance indices (STIs) for all measured traits was performed based on the Euclidean distance matrix with un-weighted pair-group method based on arithmetic averages (UPGMA) in the software NTSYS-pc ver 2.1 (Fig. 1). Cluster analysis revealed two distinct groups with respect to heat tolerance with substantial diversity among genotypes either susceptible or tolerant to heat stress (GI and GII; Fig. 1). The first group (GI) consists of 20 genotypes which are heat stress tolerant, while the remaining 20 heat-stress susceptible genotypes were clustered to group II (GII). Cophenetic correlation between ultrametric similarities of tree and similarity matrix was estimated as $r=0.76, \mathrm{P}<0.01$.

\section{Discussion}

Projection of climatic and environmental changes necessitates the need for breeding strategies that improve both yield potential and resilience to extreme weather events such as high temperatures and drought. Heat stress around sensitive stages of wheat development has been identified as a possible threat to wheat production. However, no estimates have been made to determine yield losses due to increased frequency and magnitude of high temperature stress under climate change (Stratonovitch \& Semenov, 2015).

In the current study plants of 40 Triticum aestivum genotypes were grown under favorable and heat-stressed conditions in two successive growing seasons. Sowing dates were arranged so that plants grown under heat-stressed conditions experience hot days when they reached the booting stage. All measured morphological and agronomic traits responded differently to raising temperature during booting stages. Continuous phenotypic variations for all studied traits were observed, indicating a quantitative inheritance of heat stress tolerance. Heat stress reduces plant photosynthetic capacity through metabolic limitations and oxidative damage to chloroplasts and, potentially, cell death as a result of production of elevated concentrations of ROS (reactive oxygen species), with concomitant reductions in dry matter accumulation and grain yield (Apel \& Hirt, 2004 and Farooq et al., 2011). 
TABLE 12. 100 kernel weight $(100 \mathrm{KW})$ mean performance.

\begin{tabular}{|c|c|c|c|c|c|c|c|c|c|c|c|c|c|}
\hline \multirow{2}{*}{\multicolumn{2}{|c|}{$\begin{array}{c}\text { Season }(\mathbf{S}) \\
\text { Sowing } \\
\text { Date }(\mathbf{T})\end{array}$}} & \multicolumn{4}{|c|}{$2013 / 2014$} & \multicolumn{4}{|c|}{$2014 / 2015$} & \multicolumn{3}{|c|}{ Over all Mean } & \multirow{2}{*}{$\underbrace{\mathbb{E}}_{0}$} \\
\hline & & \%ิ & 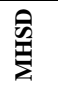 & \%ิ & 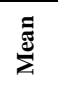 & 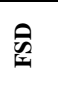 & 盆 & స్య & 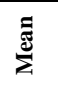 & 空 & 㟒 & \%ి & \\
\hline \multirow{40}{*}{$\begin{array}{l}\text { (0) } \\
0 \\
0 \\
0 \\
0 \\
0 \\
0 \\
0\end{array}$} & Sakha 8 & 4.45 & 3.70 & 3.70 & 3.95 & 5.98 & 4.70 & 3.48 & 4.72 & 5.21 & 4.20 & 3.59 & 4.33 \\
\hline & Sakha 69 & 3.79 & 3.15 & 3.13 & 3.35 & 4.37 & 4.22 & 3.14 & 3.91 & 4.08 & 3.69 & 3.14 & 3.64 \\
\hline & Sakha 92 & 3.62 & 2.93 & 2.36 & 2.97 & 5.04 & 4.93 & 4.87 & 4.94 & 4.33 & 3.93 & 3.62 & 3.96 \\
\hline & Sakha 93 & 4.73 & 3.76 & 3.31 & 3.93 & 4.74 & 4.66 & 4.60 & 4.67 & 4.73 & 4.21 & 3.95 & 4.30 \\
\hline & $\begin{array}{c}\text { Gemmeza } \\
7 \\
\end{array}$ & 5.27 & 3.76 & 3.44 & 4.15 & 4.49 & 4.37 & 4.26 & 4.37 & 4.88 & 4.06 & 3.85 & 4.26 \\
\hline & $\begin{array}{c}\text { Gemmeza } \\
10\end{array}$ & 4.37 & 3.19 & 2.91 & 3.49 & 4.19 & 4.17 & 4.15 & 4.17 & 4.28 & 3.68 & 3.53 & 3.83 \\
\hline & Misr 1 & 4.48 & 3.72 & 3.17 & 3.79 & 4.12 & 4.06 & 4.02 & 4.07 & 4.30 & 3.89 & 3.59 & 3.93 \\
\hline & Misr 2 & 4.98 & 4.38 & 3.84 & 4.40 & 4.00 & 3.98 & 3.96 & 3.98 & 4.49 & 4.18 & 3.90 & 4.19 \\
\hline & Giza 160 & 5.66 & 3.69 & 3.52 & 4.29 & 3.92 & 3.90 & 3.88 & 3.90 & 4.79 & 3.79 & 3.70 & 4.09 \\
\hline & Giza 165 & 5.07 & 3.25 & 3.14 & 3.82 & 3.85 & 3.83 & 3.81 & 3.83 & 4.46 & 3.54 & 3.47 & 3.82 \\
\hline & Giza 168 & 4.76 & 4.39 & 4.02 & 4.39 & 3.77 & 3.71 & 3.68 & 3.72 & 4.26 & 4.05 & 3.85 & 4.05 \\
\hline & Giza 171 & 3.95 & 3.92 & 3.08 & 3.65 & 3.65 & 3.61 & 3.53 & 3.60 & 3.80 & 3.77 & 3.31 & 3.62 \\
\hline & Seds 1 & 3.56 & 3.52 & 2.83 & 3.30 & 3.51 & 3.47 & 3.36 & 3.44 & 3.53 & 3.49 & 3.10 & 3.37 \\
\hline & Seds 4 & 3.87 & 3.66 & 3.54 & 3.69 & 3.26 & 3.02 & 2.81 & 3.03 & 3.56 & 3.34 & 3.17 & 3.36 \\
\hline & Seds 12 & 4.14 & 3.94 & 3.46 & 3.84 & 2.70 & 2.60 & 2.31 & 2.54 & 3.42 & 3.27 & 2.88 & 3.19 \\
\hline & Seds 13 & 3.86 & 3.10 & 3.03 & $\mathbf{3 . 3 3}$ & 4.11 & 3.44 & 3.17 & 3.57 & 3.98 & 3.27 & 3.10 & 3.45 \\
\hline & $\begin{array}{l}\text { Sahel } 1 \\
\end{array}$ & 4.45 & 3.46 & 3.00 & 3.63 & 4.90 & 3.71 & 3.23 & 3.94 & 4.67 & 3.58 & 3.11 & 3.79 \\
\hline & $\begin{array}{c}\text { Shandawee } \\
1\end{array}$ & 3.61 & 3.25 & 2.97 & 3.27 & 3.86 & 3.41 & 3.04 & 3.43 & 3.73 & 3.33 & 3.00 & 3.35 \\
\hline & Canada 462 & 4.37 & 3.21 & 2.97 & 3.51 & 4.82 & 3.83 & 3.38 & 4.01 & 4.59 & 3.52 & 3.17 & 3.76 \\
\hline & Canada 515 & 3.70 & 3.03 & 2.85 & 3.19 & 3.95 & 3.60 & 3.17 & 3.57 & 3.82 & 3.32 & 3.01 & 3.38 \\
\hline & Beknora & 3.81 & 3.71 & 3.09 & 3.54 & 4.45 & 4.03 & 3.22 & 3.90 & 4.13 & 3.87 & 3.15 & 3.72 \\
\hline & Debera & 3.88 & 3.78 & 2.67 & 3.44 & 4.33 & 4.03 & 2.50 & 3.62 & 4.10 & 3.90 & 2.59 & 3.53 \\
\hline & Nelen & 5.25 & 4.55 & 3.52 & 4.44 & 5.70 & 5.00 & 4.08 & 4.92 & 5.47 & 4.77 & 3.80 & 4.68 \\
\hline & Snora & 3.74 & 3.54 & 3.28 & 3.52 & 3.94 & 3.90 & 3.71 & 3.85 & 3.84 & 3.72 & 3.49 & 3.68 \\
\hline & Assiut 108 & 4.78 & 3.76 & 3.72 & 4.08 & 5.23 & 4.05 & 3.67 & 4.31 & 5.00 & 3.90 & 3.69 & 4.20 \\
\hline & Assiut 204 & 4.67 & 3.66 & 3.05 & 3.79 & 5.12 & 3.91 & 2.97 & 4.00 & 4.89 & 3.78 & 3.01 & 3.89 \\
\hline & Assiut 724 & 4.12 & 4.06 & 3.21 & 3.80 & 5.13 & 3.86 & 3.17 & 4.05 & 4.62 & 3.96 & 3.19 & 3.92 \\
\hline & MK 1-20 & 3.14 & 3.04 & 2.47 & 2.88 & 3.39 & 3.29 & 2.56 & 3.08 & 3.26 & 3.16 & 2.52 & 2.98 \\
\hline & MK 7-83 & 5.38 & 4.54 & 3.76 & 4.56 & 5.83 & 4.99 & 4.27 & 5.03 & 5.60 & 4.76 & 4.01 & 4.79 \\
\hline & L 1203 & 4.60 & 3.84 & 3.77 & 4.07 & 5.05 & 4.29 & 3.74 & 4.36 & 4.82 & 4.06 & 3.75 & 4.21 \\
\hline & L 1290 & 4.71 & 3.88 & 3.06 & 3.88 & 4.57 & 4.22 & 3.55 & 4.11 & 4.64 & 4.05 & 3.30 & 4.00 \\
\hline & L 1351 & 4.10 & 3.15 & 3.01 & 3.42 & 4.55 & 3.40 & 2.80 & 3.58 & 4.32 & 3.27 & 2.90 & 3.50 \\
\hline & L 1457 & 3.73 & 2.82 & 2.65 & 3.07 & 3.98 & 3.07 & 2.70 & 3.25 & 3.85 & 2.95 & 2.67 & 3.16 \\
\hline & L 741 & 5.04 & 3.74 & 3.12 & 3.97 & 5.49 & 3.85 & 2.81 & 4.05 & 5.26 & 3.80 & 2.96 & 4.01 \\
\hline & L 780 & 3.18 & 3.08 & 2.30 & 2.85 & 3.43 & 3.33 & 2.42 & 3.06 & 3.30 & 3.20 & 2.36 & 2.95 \\
\hline & L 887 & 3.84 & 3.72 & 3.14 & 3.56 & 4.09 & 3.49 & 2.93 & 3.50 & 3.96 & 3.60 & 3.03 & 3.53 \\
\hline & line 1 & 4.46 & 4.32 & 2.89 & 3.89 & 4.91 & 4.77 & 2.63 & 4.10 & 4.68 & 4.54 & 2.76 & 3.99 \\
\hline & line 3 & 3.88 & 3.62 & 2.82 & 3.44 & 4.33 & 3.92 & 3.26 & 3.83 & 4.10 & 3.77 & 3.04 & 3.64 \\
\hline & line 4 & 3.83 & 3.43 & 3.06 & 3.44 & 4.08 & 3.68 & 3.38 & 3.71 & 3.95 & 3.55 & 3.22 & 3.57 \\
\hline & line 5 & 4.98 & 2.98 & 2.47 & 3.47 & 5.43 & 3.21 & 2.68 & 3.77 & 5.20 & 3.10 & 2.57 & 3.62 \\
\hline \multicolumn{2}{|c|}{ Mean } & 4.30 & 3.61 & 3.13 & 3.68 & 4.41 & 3.89 & 3.37 & 3.89 & 4.35 & 3.75 & 3.25 & 3.78 \\
\hline \multicolumn{2}{|c|}{ LSD' $_{0.05}(\mathrm{~S})$} & 0.10 & & & & \multicolumn{4}{|c|}{ LSD' $_{0.05}(\mathrm{G})$} & \multicolumn{3}{|l|}{0.10} & \\
\hline \multicolumn{2}{|c|}{ LSD' $_{0.05}(\mathrm{~T})$} & 0.12 & & & & \multicolumn{4}{|c|}{ LSD' $_{0.05}\left(\mathrm{G}^{*} \mathrm{~S}\right)$} & 0.14 & & & \\
\hline \multirow{2}{*}{\multicolumn{2}{|c|}{ LSD' $_{0.05}\left(\mathrm{~S}^{*} \mathrm{~T}\right)$}} & NS & & & & \multicolumn{4}{|c|}{ LSD' $_{0.05}(\mathrm{G} * \mathrm{~T})$} & 0.17 & & & \\
\hline & & & & & & \multicolumn{4}{|c|}{ LSD' $_{0.05}(\mathrm{G} * \mathrm{~S} * \mathrm{~T})$} & 0.25 & & & \\
\hline
\end{tabular}

Egypt. J. Agron. 38, No. 3 (2016) 


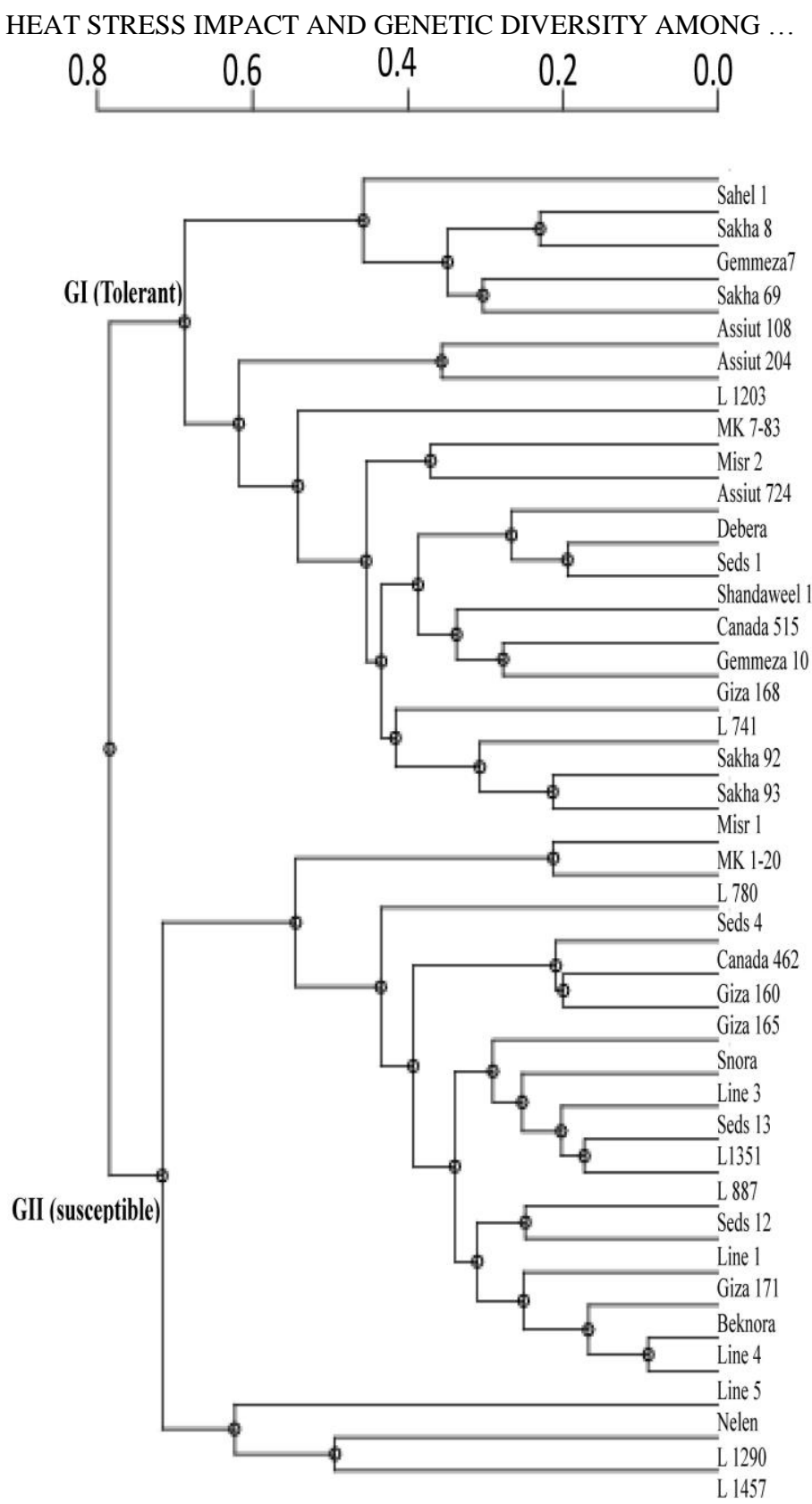

407 
The dramatic decrease in all studied yield and yield-contributing traits under heatstressed conditions might be due to the inhibition of photosynthesis as one of the most apparent effects of heat stress on photosynthetic tissues. High temperatures damage the OEC (oxygen evolving complex) of PSII (photosystem II), reduce Rubisco activity, and cause disorganization of the thylakoid membranes. Besides, the reduction of photosynthetic capacity during reproductive transition ultimately results in reduction of parental resources available for reproduction (Gorantla et al., 1984 ; Law \& Crafts-Brandner, 1999 and Strasser, 1997). Furthermore, flowering plants are highly sensitive to temperature stresses during the reproductive phase, which covers floral initiation to seed maturity, with even a single hot day sometimes being determinant to reproductive success. (Zinn et al., 2010).

Cluster analysis revealed two distinct groups based on heat stress tolerance. Cophenetic correlation between ultrametric similarities of tree and similarity matrix was found to be relatively high $(\mathrm{r}=0.76, \mathrm{P}<0.01)$, suggesting that the cluster analysis strongly represents the similarity matrix.

In conclusion, screening the primary gene pool of wheat for heat tolerance identified six genotypes represent variable degrees of tolerance to heat stress. The current study has demonstrated that screening the primary gene pool of wheat is a useful source for improving quantitative heat stress tolerance related traits. Besides, our data could serve as a vitally important tool in improving barley breeding for heat stress conditions by exploiting the identified heat tolerant genotypes in wheat breeding programs.

Finally we could conclud that the successful process of wheat breeding based on the knowledge of characteristics of genotypes, environment and their interaction. As in current study we could found that there are some new promising genotypes gaining the ability to be tolerant to sever heat stress during post anthesis and grainfilling phases stage depend on the purpose of breeding programs. For example if the program aims to produce high biomass yield some lines proved its ability such as L1457, Assiut 204 and L887. Also we can conclude that, genotypes which have been developed in hot regions can produce high yield in heat stress conditions such as cultivar Bicnora. In contrary also genotypes which developed in regions without a biotic stress face a big reduction when transferred to stress regions such as cultivar Gemmeza 10 which recorded the highest reduction in $\mathrm{HI}$ in sever conditions.

Acknowledgments: The authors gratefully acknowledge to Prof. Dr. M. El-Morshidy as he provided us with the promising lines and land races which used in this study. Also authors gratefully thanks the staff of Agronomy Department Farm at Assiut University for their excellent technical assistance.

\section{References}

Apel, K. and Hirt, H. (2004) Reactive oxygen species: metabolism, oxidative stress, and signal transduction. Annual Review of Plant Biology, 55, 373-399.

Egypt. J. Agron. 38, No. 3 (2016) 
Birol, T.A. and Necmettin, C.E.L. (2011) Determination of seed yield and some yield components through path and correlation analyses in many sixrowed barley (Hordeum vulgare conv. hexastichon). African Journal of Agricultural Research, 6 (21), 49024905.

Donald, C.M. and Hamblin, J. (1976) The biological yield and harvest index of cereals as agronomic and plant breeding criteria. Adv. Agron. 28, 361-405.

Elhani, S., Martos, V., Rharrabti, Y., Royo, C. and García del Moral, L.F (2007) Contribution of main stem and tillers to durum wheat (Triticum turgidum L. var. durum) grain yield and its components grown in Mediterranean environments. Field Crops Research, 103, 25-35.

FAOSTAT (2014) Food and Agriculture Organization of the United Nations. http://faostat.fao.org.

Farooq, M., Bramley, H., Palta, J.A. and Siddique, K.H.M. (2011) Heat stress in wheat during reproductive and grain-filling phases. Critical Reviews in Plant Sciences, 30, 1-17.

Fernandez, G.C.J. (1992) Effective selection criteria for assessing stress tolerance. In : Proceedings of the International Symposium on Adaptation of Vegetables and Other Food Crops in Temperature and Water Stress, Kuo, C.G. (Ed.), Publication. Tainan Taiwan.

Fischer, R.A. and Byerlee, D.B. (1991) Trends of wheat production in the warmer areas: major issues and economic considerations. In: Wheat for the Non-traditional Warm Areas. Proceeding Conference. CIMMYT, Iguazu. pp 3-27.

Fokar, M., Blum, A. and Nguyen, H.T. (1998) Heat tolerance in spring wheat. II. Grain filling. Euphytica, 104 (1), 9-15.

Godfray, H.C.J., Beddington, J.R., Crute, I.R., Haddad, L., Lawrence, D., Muir, J.F., Pretty, J., Robinson, S., Thomas, S.M. and Toulmin, C. (2010) Food security: the challenge of feeding 9 billion people. Science , 327, 812-818.

Gorantla, M., Babu, P.R., Lachagari, V.B.R., Reddy, A.M.M., Wusirika, R., Bennetzen, J.L., Gounaris, K., Brain, A.R.R., Quinn, P.J. and Williams, W.P. (1984) Structural reorganization of chloroplast thylakoid membranes in response to heat-stress. Biochimica et Biophysica Acta. 766, 198-208.

Joshi, A.K., Chand, R., Arun, B., Singh, R.P. and Ortiz, R. (2007) Breeding crops for reduced-tillage management in the intensive, rice-wheat systems of South Asia. Euphytica, 153 (1-2), 135-151.

Law, R.D. and Crafts-Brandner, S.J. (1999) Inhibition and acclimation of photosynthesis to heat stress is closely correlated with activation of ribulose-1,5-bisphosphate carboxylase/oxygenase. Plant Physiology, 120, 173-181. 
Liu, G.F., Yang, J., Xu, H.M., Hayat, Y. and Zhu, J. (2008) Genetic analysis of grain yield conditioned on its component traits in rice (Oryza sativa L.). Aust. J. Agric. Res. 59, 180-195.

Maestri, E., Klueva, N., Perrotta, C., Gulli, M., Nguyen, H.T. and Marmiroli, N. (2002) Molecular genetics of heat tolerance and heat shock proteins in cereals. Plant Molecular Biology, 48, 667-681.

Martincic, J., Bede, M. and Maric, S. (1996) Connection between ear length and kernel yield and quality in winter wheat varieties. Proc. $10^{\text {th }}$ Inter. Cereal Breed Congr., Port Caras. p. 111.

Nei, M. and Li, W.H. (1979) Mathematical model for studying genetic variation in terms of restriction endonucleases. Proc. Natl. Acad. Sci. USA, 76, 5269-5273.

Parry, M.A.J., Reynolds, M.P., Salvucci, M.E., Raines, C., Andralojc, P.J., Zhu, X.G., Price, G.D., Condon, A.G. and Furbank, R.T. (2011) Raising yield potential of wheat. II. Increasing photosynthetic capacity and efficiency. Journal of Experimental Botany, 62, 453-467.

Reynolds, M.P., Bonnett, D., Chapman, S.C., Furbank, R.T., Manès, Y., Mather, D.E. and Parry, M.A.J. (2011) Raising yield potential of wheat. I. Overview of a consortium approach and breeding strategies. Journal of Experimental Botany, 62, $439-452$.

Rohlf, F.J. (2000) NTSYS-pc, Version 2.1d. Exeter Software, Setauket, N.Y.

Said, A.A., Hamada, A. and Youssef, M. (2015) Assessment of heat tolerance in bread wheat using some agronomic traits and SRAP markers. Egypt. J. Plant Breed. 19 (3), $979-994$.

Sharma, R.C. (1993) Selection for biomass yield in wheat. Euphytica, 70 (1), 35-42.

Steel, G.D. and Torrie, J. H. (1981) "Principles and Procedures of Statistics" ( $2^{\text {nd }}$ ed.) McGraw-Hill Book Company. Inc. N. Y. xxi-633pp.

Strasser, B.J. (1997) Donor side capacity of Photosystem II probed by chlorophyll a fluorescence transients. Photosynthesis Research, 52, 147-155.

Stratonovitch, P. and Semenov, M.A. (2015) Heat tolerance around flowering in wheat identified as a key trait for increased yield potential in Europe under climate change. J. Exp. Bot. 66, 3599-3609.

Suzuki, N., Rivero, R.M., Shulaev, V., Blumwald, E. and Mittler, R. (2014) Abiotic and biotic stress combinations. New Phytol. 203, 32-43.

Wahid, A., Gelani, S., Ashraf, M. and Foolad, M.R. (2007) Heat tolerance in plants: an overview. Environmental and Experimental Botany, 61, 199-223.

Wang, W.X., Vinocur, B. and Altman, A. (2003) Plant responses to drought, salinity and extreme temperatures: towards genetic engineering for stress tolerance. Planta, 218, $1-14$

Egypt. J. Agron. 38, No. 3 (2016) 
Wardlaw, I.F., Blumenthal, C., Larroque, O. and Wrigley, C.W. (2002) Contrasting effects of chronic heat stress and heat shock on kernel weight and flour quality in wheat. Funct. Plant Biol. 29 (1), 25-34.

Weikai, Y. and Hunt, L.A. (2001) Interpretation of genotype x environment interaction for winter wheat yield in Ontario. Crop Science , 41, 19-25.

Yang, J., Sears, R.G., Gill, B.S. and Paulsen, G.M. (2002) Quantitative and molecular characterization of heat tolerance in hexaploid wheat. Euphytica ,126, 275-282.

Zinn, E.K., Tunc-Ozdemir, M. and Harper, J.F. (2010) Temperature stress and plant sexual reproduction: uncovering the weakest links. J. Exp. Bot. 61 (7), 1959-1968.

(Received 4/ 9/2016;

accepted $5 / 10 / 2016)$ 


\title{
تاثير الاجهاد الدرارى والتنوع الوراثى على مجموعة من التراكيب الوراثية لقمح الخبز
}

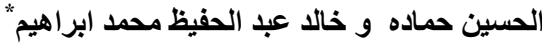

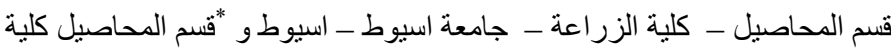

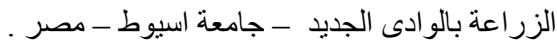

يعتبر القمح من المحاصيل واسعة الانتشار خاصة فى الدول النامية ولكن حديثا

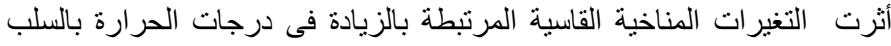

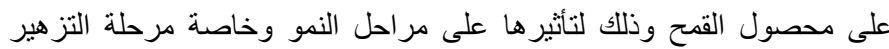
و امتلاء الحبوب. تعتبر صفة المقاومة للحر ارة من الصفات المعقدة حيث أنها تتاثر

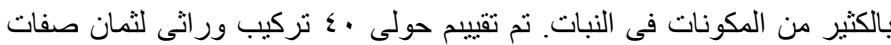

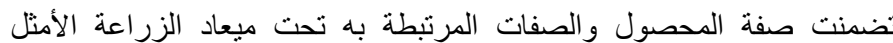

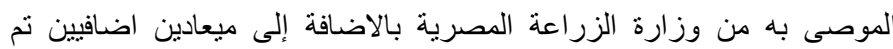
اختيارهم بحيث يتعرض النبات لظروف الحرارة الزّاعة القاسية فى مرحلة الازهار وامتلاء الحبوب.

أظهرت جميع الصفات قبد الدراسة استجابة معنوية سو اء لمو اعبد الزراعة او

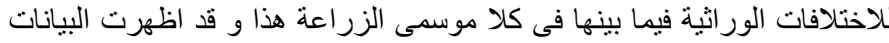
الماخوذة دلالة على أن هذه الصفات قد تكون تحت تاثثير العديد من الجينات.

أظهر تحليل التباين تاثير ا عالى المعنوية للتفاعل بين التراكيب الوراثية والبيئة

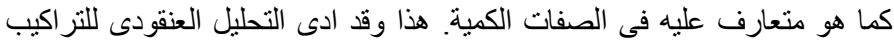

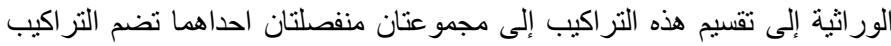

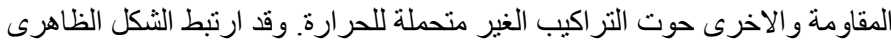

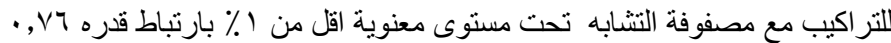
مما يرجح ان التحليل العنقودى نجح بقوة فى تقسيم التر اكيب والتمبيز بينها. 